\title{
Hubble Space TelescopeObservations of NGC 6240: A Case Study of an Ultraluminous Infrared Galaxy with Obscured Activity
}

\section{Citation}

Gerssen, Joris, Roeland P. van der Marel, David Axon, J. Christopher Mihos, Lars Hernquist, and Joshua E. Barnes. 2004. "Hubble Space TelescopeObservations of NGC 6240: A Case Study of an Ultraluminous Infrared Galaxy with Obscured Activity." The Astronomical Journal 127 (1): 75-89. https://doi.org/10.1086/380223.

\section{Permanent link}

http://nrs.harvard.edu/urn-3:HUL.InstRepos:41381841

\section{Terms of Use}

This article was downloaded from Harvard University's DASH repository, and is made available under the terms and conditions applicable to Other Posted Material, as set forth at http:// nrs.harvard.edu/urn-3:HUL.InstRepos:dash.current.terms-of-use\#LAA

\section{Share Your Story}

The Harvard community has made this article openly available.

Please share how this access benefits you. Submit a story.

\section{Accessibility}


Astronomical Journal, Jan 2004, in press

\title{
Hubble Space Telescope Observations of NGC 6240: a Case Study of an Ultra-Luminous Infrared Galaxy with Obscured Activity
}

\author{
Joris Gerssen, Roeland P. van der Marel \\ Space Telescope Science Institute, 3700 San Martin Drive, Baltimore, MD 21218 \\ David Axon \\ Department of Physics, Rochester Institute of Technology, 84 Lomb Memorial Drive, Rochester, \\ NY 14623 \\ J. Christopher Mihos ${ }^{1}$ \\ Department of Astronomy, Case Western Reserve University, 10900 Euclid Avenue, Cleveland, \\ $\mathrm{OH} 44106$ \\ Lars Hernquist \\ Harvard University, Center for Astrophysics, 60 Garden Street, MS-51, Cambridge MA 02138 \\ Joshua E. Barnes \\ University of Hawaii, Institute for Astronomy, 2680 Woodlawn Drive, Honolulu, HI 96822
}

\footnotetext{
${ }^{1}$ Research Corporation Cottrell Scholar and NSF CAREER Fellow
} 


\begin{abstract}
We present results from a Hubble Space Telescope (HST) study of the morphology and kinematics of NGC 6240. This merging galaxy with a double nucleus is one of the nearest and best-studied ultraluminous infrared galaxies. HST resolves both nuclei into seperate components. The distance between the northern and southern optical/near-infrared components is greater than that observed in radio and X-ray studies, arguing that even in K-band we may not be seeing all the way through the dust to the true nuclei.

The ionized gas does not display rotation around either of the nuclei, or equilibrium motion in general. There is a strong velocity gradient between the nuclei, similar to what is seen in CO data. There is no such gradient in our stellar kinematics. The velocity dispersion of the gas is larger than expected for a cold disk. We also map and model the emission-line velocity field at an off-nuclear position where a steep velocity gradient was previously detected in ground-based data. Overall, the data indicate that line-of-sight projection effects, dust absorption, non-equilibrium merger dynamics, and the possible influence of a wind may be playing an important role in the observed kinematics.

Chandra observations of hard X-rays have shown that both of the nuclei contain an Active Galactic Nucleus (AGN). The HST data show no clear sign of the two AGNs: neither continuum nor narrow-band imaging shows evidence for unresolved components in the nuclei, and there are no increased emission line widths or rapid rotation near the nuclei. This underscores the importance of X-ray data for identifying AGNs in highly dust-enshrouded environments.
\end{abstract}

Subject headings: galaxies: interactions — galaxies: kinematics and dynamics galaxies: nuclei — galaxies: starburst — galaxies: structure.

\title{
1. Introduction
}

Ultraluminous Infrared Galaxies (ULIRGs) have infrared luminosities in excess of $\sim 10^{12} \mathrm{~L}_{\odot}$. These galaxies were first discovered by the IRAS satellite (e.g., Soifer, Neugebauer \& Houck 1987). They are usually associated with mergers and interactions of galaxies (e.g., Sanders \& Mirabel 1996) and their infrared emission is powered a combination of a nuclear starburst and an embedded AGN (e.g. Genzel et al. 1998). It is believed that the ULIRGs observed locally are related in an evolutionary sense to the onset of quasar activity (Sanders et al. 1988), the formation of (elliptical) galaxies (Kormendy \& Sanders 1992), the sub-millimeter sources observed with SCUBA (Smail, Ivison, \& Blain 1997), and the many obscured AGNs detected with Chandra (Barger et al. 2001).

NGC 6240 at a distance of $104 \mathrm{Mpc}$ (assuming $H_{0}=70 \mathrm{~km} \mathrm{~s}^{-1} \mathrm{Mpc}^{-1}$, both here and throughout this paper) has $L_{\mathrm{IR}}=10^{11.8} \mathrm{~L}_{\odot}$, just below the canonical ULIRG luminosity boundary 
of $10^{12} \mathrm{~L}_{\odot}$. Nonetheless, it is generally regarded both as a bona fide ULIRG, and as one of the nearest and best studied examples of the class (Genzel et al. 1998). The large-scale optical morphology of NGC 6240 is highly distorted and shows the tidal tails indicative of an ongoing merger (Keel 1990; see also Figure 1). Ground-based imaging at both optical (Schulz et al. 1993) and near-infrared wavelengths (Doyon et al. 1994) has revealed a double nucleus with a separation of $\sim 1.8^{\prime \prime}=0.88 \mathrm{kpc}$. Radio continuum images also show the double nucleus (Carral, Turner, \& Ho 1990; Eales et al. 1990; Beswick et al. 2001), as do [Fe II] images at 1.64 $\mu$ (van der Werf et al. 1993).

The infrared emission from NGC 6240 comes from dust heating. Mid-infrared line-ratio measurements with ISO show that the heating is due partly to a nuclear starburst triggered by the interaction, and partly due to an AGN continuum (Genzel et al. 1998). The optical emission of NGC 6240 is characterized by a LINER-type spectrum and the width of the $\mathrm{H} \alpha+[\mathrm{NII}]$ emission lines increases strongly towards the double nucleus (Keel 1990). The presence of an AGN component in NGC 6240 was confirmed by the detection of hard X-rays with ASCA (Iwasawa \& Comastri 1998) and BEPPO-SAX (Vignati et al. 1999). Interestingly, subsequent Chandra observations showed that there is actually an AGN associated with each of the two nuclei of NGC 6240 (Komossa et al. 2003). It is not necessarily surprising to find two AGNs in a merger of two galaxies, given that most galaxies are believed to harbor central black holes (e.g., Kormendy \& Gebhardt 2001) and given that mergers have been implicated as triggers for AGN activity. However, NGC 6240 is the first galaxy for which the presence of two supermassive black holes has been convincingly demonstrated.

The stellar velocity field in the region of the double nucleus has been studied from the ground by Tecza et al. (2000). The velocity field of molecular CO gas was studied by Tacconi et al. (1999). Neither of these studies found a tell-tale signature of one of the black holes in NGC 6240 in the form of unusually rapidly moving stars or gas. However, this is not surprising given that the spatial resolution of Hubble Space Telescope is generally required to detect the gravitational influence of super-massive black holes in galaxies.

NGC 6240 is of considerable importance as a nearby ULIRG proto-type. Its structure has therefore been studied in great detail at many wavelengths. However, ground-based studies are generally limited to resolutions near $\sim 1^{\prime \prime}=0.49 \mathrm{kpc}$. By contrast, HST can probe down to scales of $\sim 0.05^{\prime \prime}=25 \mathrm{pc}$. We present here the results of an HST study of the morphology and kinematics of NGC 6240 at optical wavelengths, with the Second Wide Field and Planetary Camera (WFPC2) and Space Telescope Imaging Spectrograph (STIS), respectively. Previous HST studies of NGC 6240 were restricted to pre-COSTAR U-band imaging with the Faint Object Camera (FOC) by Barbieri et al. (1993) and Near-Infrared Camera and Multi-Object Spectrometer (NICMOS) imaging by Scoville et al. (2000) and van der Werf (2001). The U-band data showed multiple components in the nuclear region, probably due to patchy dust obscuration. The NICMOS data confirmed the double nucleus.

ULIRGs, and galaxy mergers in general, tend to be more dusty than normal galaxies. This can complicate investigations into their structure. Near-IR or mid-IR observations are definitely best suited to penetrate the dust. On the other hand, the observational capablities at these wavelengths are not always comparable to what can be achieved at optical wavelengths. For 
example, HST has an optical spectrograph, but no near-IR spectrograph. Of course, optical observations are best restricted to those galaxies for which one has a chance to penetrate the dust. Ground-based optical observations of NGC 6240 have shown the same double nuclues structure seen in radio and X-ray data. This suggests that optical observations may be seeing far enough through the dust to be able to probe the regions where the radio and X-ray emission are produced. With this in mind, we have used HST to study NGC 6240 with the following goals:

(1) Improve our understanding of the nuclear structure and morphology of NGC 6240. Groundbased observations have found that the separation between the two nuclei of NGC 6240 is somewhat wavelength dependent (Schulz et al. 1993), which indicates that dust absorption is not negligible. The spatial resolution of HST, combined with the availability of data in various bands, allows us to study the distribution and optical depth of the dust. Comparison of the observed morphology to that at radio and X-ray wavelengths provides insight into the extent to which the AGN components of NGC 6240 are, or are not, obscured at optical and near-infrared wavelengths.

(2) Search for tell-tale signs of the AGN components in NGC 6240. For example, unresolved continuum emission might point to optical synchrotron emission from one of the AGNs. Increases in stellar or gaseous motions can pin-point the gravity of a black hole, and if detected, might allow measurements of the masses of the black holes in NGC 6240.

(3) Examine the extent to which the kinematical characteristics of NGC 6240 are consistent with relaxed quasi-equilibrium motions. This is relevant for a variety of issues. For example, Bland-Hawthorn, Wilson \& Tully (1991) suggested that the large-scale velocity field can be decomposed into the contributions of two rotating disks. Similarly, Tecza et al. (2000) suggested that the small-scale velocity field near the double nucleus of the system can be modeled as two rotating nuclear disks. Conversely, Tacconi et al. (1999) fit the nuclear CO velocity field with a single disk centered between the nuclei. Lester \& Gaffney (1994) used the stellar velocity dispersion to estimate the mass of NGC 6240, and Doyon et al. (1994) used it to place NGC 6240 on the fundamental plane of elliptical galaxies.

In Section 2 we present WFPC2 imaging of NGC 6240 in the broad B, V, and I-bands, as well as narrow-band imaging of $\mathrm{H} \alpha+[\mathrm{NII}]$ emission. In Section 3 we study the small-scale velocity field of NGC 6240 using STIS Ca triplet absorption-line spectroscopy and $\mathrm{H} \alpha+[\mathrm{NII}]$ emission line spectroscopy of the nuclear region. We also use $\mathrm{H} \alpha+[\mathrm{NII}]$ emission line spectroscopy to map and model the gas velocity field at the off-nuclear position where a steep velocity gradient was previously detected in ground-based data (Bland-Hawthorn et al. 1991). Conclusions are presented in Section 4. Preliminary results of our project were discussed previously in Gerssen et al. (2001). Also, some of our WFPC2 data have already been used in archival studies by other groups. Our $\mathrm{H} \alpha+[\mathrm{NII}]$ image was discussed by Lira et al. (2002) and Komossa et al. (2003) in the context of X-ray observations of NGC 6240. Our broad-band images were used by Pasquali, de Grijs \& Gallagher (2003) in a study of the star cluster population of NGC 6240. 


\section{WFPC2 Imaging}

\subsection{Observations and Data Reduction}

Broad- and narrow-band images of NGC 6240 were obtained in March 1999 with the WFPC2 on board of HST in the context of program GO-6430 (PI: van der Marel). The WFPC2 is described in detail by, e.g., Biretta et al. (2001). The observations were obtained in a single 'visit' of four spacecraft orbits. The WFPC2 has four chips, each with $800 \times 800$ pixels. All exposures were obtained with the galaxy centered on the PC chip to obtain the highest spatial resolution (pixel size $0.046^{\prime \prime}$ ). Figure 1 shows the PC field of view overlaid on an NGC 6240 image extracted from the Digitized Sky Survey.

The observing $\log$ is presented in Table 1 . Broad band observations were obtained with the F450W, F547M and F814W filters (roughly Johnson $B, V$ and $I$ ). Narrow band observations were obtained using the F673N and the F658N filters. At the redshift of NGC $6240(z=0.02427)$ the $\mathrm{H} \alpha+[\mathrm{NII}]$ lines are shifted into the wavelength range of the F673N ('on-band') filter. NGC 6240 is emission-line free in the F658N ('off-band') filter.

The images were calibrated by the HST calibration 'pipeline' maintained by the Space Telescope Science Institute (STScI). The standard reduction steps include bias subtraction, dark current subtraction and flat-fielding, as described in detail by Holtzman et al. (1995a). Two or three different exposures were taken with each filter, each with the target shifted by a small integer number of pixels. The different exposures for each filter were aligned, and then combined with simultaneous removal of cosmic rays and chip defects. This yields one final image per filter. To obtain an image of the $\mathrm{H} \alpha+[\mathrm{NII}]$ emission we aligned the off-band image with the on-band image, scaled it to fit the on-band image at radii where the ionized gas flux is negligible, and subtracted it from the on-band image.

The count-rates in the broad-band F450W, F547M and F814W images were calibrated to magnitudes in the Johnson $B, V$ and $I$ bands, respectively, as described in Holtzman et al. (1995b). The count-rates in the F673N image were calibrated to erg $\mathrm{cm}^{-2} \mathrm{~s}^{-1}$ using calculations with the SYNPHOT package in IRAF.

\subsection{Global Morphology}

Figure 2 shows a composite color image of the three broad band images for the full PC field of view, with the $\mathrm{H} \alpha+[\mathrm{NII}]$ image overlaid in yellow. The broad-band data show a complex morphology, consistent with the assumed merger nature of NGC 6240. A wide dust lane is visible against the main body of the galaxy, which was studied previously by Keel (1990) using unsharp masking. The WFPC2 image shows additional filamentary dust structures on smaller scales. These extend into the very central region and (partly) cover the nuclear region.

Heckman, Armus \& Miley $(1987,1990)$ found that a morphologically spectacular emission-line nebula is associated with NGC 6240. They interpreted this as evidence for a 'superwind'. The 
kinetic energy provided by supernovae and winds from massive stars in a central starburst drives a large-scale outflow that can shock-heat and accelerate ambient interstellar and circumgalactic gas. Figure 2 reveals the intricate nature of the superwind at high spatial resolution. The emission-line filaments (yellow) appear to trace out a bipolar outflow pattern, with an axis aligned roughly E-W, perpendicular to the dust lane and the line connecting the two nuclei. This morphology is similar to that seen in other starburst superwind galaxies. The curved filament at the top of the image may be due to expelled gaseous material that is now falling back towards the central parts of the galaxy. Extended X-ray (0.5-1.5 keV) images (Lira et al. 2002; Komossa et al. 2003) have a morphology similar to the $\mathrm{H} \alpha+[\mathrm{NII}]$ image, as would be expected if the origin of the filaments is a superwind. In particular, Komossa et al. (2003) report several X-ray loops and knots that have a corresponding bright structure in our WFPC2 gas emission line image. The connection between the X-ray observations and our $\mathrm{H} \alpha+[\mathrm{NII}]$ image was already discussed in detail by Lira et al. (2002) and Komossa et al. (2003), so we will not further discuss it here.

\subsection{Nuclear Structure}

Figure 3 shows the central region of NGC 6240 at high contrast. Figure 3a shows a true-color image of the broad-band WFPC2 data, and Figure 3b shows a gray scale image of the $\mathrm{H} \alpha+[\mathrm{NII}]$ data. Previous studies of NGC 6240 have revealed that it has a double nucleus. The new HST images show that the central structure is in fact more complicated. Instead of two, there are three distinct nuclear components, both in broad-band and in $\mathrm{H} \alpha+[\mathrm{NII}]$ gas emission. We will refer to these components as N1, N2 and N3, as indicated in Figure 3a. N1 corresponds to the northern nucleus that had been identified from ground-based data, while N2 and N3 jointly correspond to the southern nucleus identified from ground-based data. The distance between N2 and N3 is $0.45^{\prime \prime}$, which explains why these components were not spatially resolved in arcsec-resolution ground-based data.

We determined the magnitudes of the nuclear components in each of the three available broad-band filters. For each nucleus we used a circular aperture with a radius of 4 pixels $\left(0.182^{\prime \prime}\right)$. The results are listed in Table 2. The magnitudes themselves are not of particular interest, since they depend strongly on the adopted aperture size. The implied colors are of more direct physical interest. N2 is the reddest of the three nuclei, as is evident also from Figure 3a. The centroid of the combined light of $\mathrm{N} 2$ and N3 varies as a function of wavelength, because N2 and N3 do not have the same color. In the $B$-band the centroid is close to N3, while in the $I$-band it is between N2 and N3. This explains the ground-based data of Schultz et al. (1993), which show that the separation between the northern and the southern nucleus of NGC 6240 decreases with increasing wavelength.

We analyzed a NICMOS K-band image from the HST archive (obtained February 1998, program GO-7219, PI: Scoville) in order to further improve our understanding of the nuclear structure. A gray-scale image of the $K$-band data is shown in Figure 3c, at the same scale and orientation as Figures 3a,b. This K-band image was discussed previously, in combination with NICMOS J and H-band data, by Scoville et al. (2000). The $K$-band morphology shows only 
two main components, unlike the optical images. The brightest pixel in the elongated southern component of the $K$-band image coincides with N2, confirming that N2 is indeed the true center of the southern component. The N3 component cannot be identified on the $K$-band image, although its location coincides with the extension of the southern component. N3 is therefore most likely either an HII region or an artifact resulting from the highly patchy dust structure in the nucleus of NGC 6240, rather than a physically distinct nuclear component. Note that the FWHM of the point spread function (PSF) is much larger for the K-band data (0.19 arcsec) than for, e.g., the V-band data (0.06 arcsec; these PSF FWHM values were calculated with the TinyTim software of Krist \& Hook 2001). This is (at least part of) the reason that the K-band morphology looks smoother than the optical morphology. However, the K-band FWHM is much less than the distance between N2 and N3 $\left(0.45^{\prime \prime}\right)$. The fact that N3 cannot be identified as a distinct peak in the K-band is therefore not an artifact of limited resolution. To facilitate a comparison with the optical results we determined the $K$ band magnitudes in the same three apertures used for the WFPC2 photometry. The results are listed in Table 2.

It is interesting to note that the northern $\mathrm{N} 1$ component in the $K$-band image is much more elongated than in the $I$-band. It appears that the southern extension to the N1 component that is visible in the K-band data (indicated as $\mathrm{N}^{\prime}$ in Figure 3c) could in fact be a separate component. This suggestion is strengthened by a Keck adaptive optics K-band image of the central region of NGC 6240, obtained by C. Max and shown in Schneider (2002). That image has slightly higher spatial resolution than the NICMOS image. If indeed $\mathrm{N} 1^{\prime}$ is a separate component it must have high extinction, given that it does not show up in the I-band data.

The amount of dust absorption towards the nuclear components can be estimated if one assumes that the dust is in front of the nuclei, and if one has estimates of the intrinsic colors. The largest color baseline line of the data is provided by the $B-K$ colors. The $A_{V}$ can be calculated from the color excess $E_{B-K}=(B-K)_{\text {obs }}-(B-K)_{\text {intrinsic. The extinction law of }}$ Rieke \& Lebofsky (1985) yields $A_{V}=0.825 E_{B-K}$. The average $B-K$ colors for late-type spiral, early-type spiral and elliptical galaxies are 3.18, 3.75 (de Jong 1996) and 4.15 (Persson, Frogel \& Aaronson 1979; Carollo et al. 1997) respectively. If any of these are taken as estimates of the intrinsic colors of the NGC 6240 nuclei, then this yields three estimates of $A_{V}$. Table 2 lists the average of the estimates thus obtained. The error bars indicate the range spanned by the calculated values. These derived $A_{V}$ estimates are lower limits because young stellar populations can be much bluer than the global colors of normal galaxies. Knowledge of the stellar population age near the center of NGC 6240 is available from other work, but unfortunately this does not help much in constraining the intrinsic $B-K$ color. Tecza et al. (2000) used the CO 2-0 absorption bandhead equivalent width to estimate the burst age at $20 \mathrm{Myr}$. At this age, the 'Starburst99' software of Leitherer et al. (1999; available at http://www.stsci.edu/science/starburst99/) predicts for a single-burst population of solar metallicity and Salpeter IMF that $B-K \approx 1.6$. On the other hand, for a $10 \mathrm{Myr}$ old population the predicted color is $B-K \approx 3.3$ and for a $5 \mathrm{Myr}$ old population it is $B-K \approx 0.3$. It is unclear whether the population of NGC 6240 is understood well enough to confidently choose among these ages and colors. The bluest that the population could reasonably be is $B-K \approx 0.0$, in which case the $A_{V}$ values in Table 2 would need to be increased by $\sim 3$. Because of the large uncertainties in our estimates of $A_{V}$ that already result from our 
ignorance of the intrinsic $B-K$ colors, we have not attempted to correct for the differences in the PSF between the $B$ - and $K$-band data. These corrections are not expected to be large because we have chosen to adopt an aperture size for the photometry that exceeds the PSF FWHM in both bands. The values $V_{\text {corr }}$ in Table 2 list the $\mathrm{V}$-band magnitudes of the nuclei corrected for our estimated dust absorption, $V_{\text {corr }}=V-A_{V}$. The results indicate that N2 is the intrinsically brightest nuclear component, as already suggested by the K-band image.

Tecza et al. (2000) recently estimated the dust absorption towards NGC 6240 from near-IR spectra in the $K$-band. Assuming, as do we, that the dust resides in a foreground screen they find $A_{V}=1.6$ for the northern nucleus. This is somewhat lower than our value $A_{V}(N 1)=2.35$. For the southern nucleus they find $A_{V}=5.8$, which exceeds the values $A_{V}(N 2)=4.75$ and $A_{V}(N 3)=3.65$ inferred here. This discrepancy is presumably due at least in part to the different spatial resolution of their data $\left(0.8^{\prime \prime}-1.0^{\prime \prime}\right)$. It is clear from Figure 3 that the dust absorption in NGC 6240 is quite filamentary and spatially variable, so $A_{V}$ estimates for different spatial regions need not necessarily agree. Tecza et al. point out that $A_{V}$ would be higher by a factor $\sim 2.5$ if the dust were well-mixed, instead of in the foreground. If this is indeed the case, this would bring our estimates more in line with other estimates in the literature. We also caution that our measurements only probe to the depth at which the nuclei become optically thick in $\mathrm{K}$. If we are not seeing all the way into the nuclei, measurements at longer wavelengths will result in higher inferred values of $A_{V}$. For example, Rieke et al. (1985) adopted $A_{V} \approx 15$ based on the continuum levels in $K$ and $L$ spectrophotometry. Genzel et al. (1998) listed extinction values for a number of ULIRGs based on line ratios obtained from ISO SWS data, yielding typical $A_{V}$ values ranging from 5 to 45 , and a value specifically for NGC 6240 of $A_{V} \geq 5$. However, it should be kept in mind that all these estimates refer to considerably larger spatial regions in NGC 6240 than the estimates derived here from the HST data. On smaller scales, Vignati et al. (1999) estimated from BeppoSAX X-ray observations of NGC 6240 that the HI column density is about $10^{21}$ to $10^{22}$ atoms $\mathrm{cm}^{-2}$, from which they infer $A_{V} \sim 10$.

The morphology of the $\mathrm{H} \alpha+[\mathrm{NII}]$ emission from the nuclear region (Figure $3 \mathrm{~b}$ ) is similar to what is seen in the optical broad-band images. The three components N1, N2 and N3 can be clearly distinguished. The last column in Table 2 reports the $\mathrm{H} \alpha+[\mathrm{NII}]$ emission line fluxes determined in the same apertures as used for the broad band images. The N1 component contains the most flux.

For all images we estimated the sizes of the nuclear components using the tools in the IRAF task imexamine. In each waveband we determined the FWHM of an azimuthally averaged radial profile centered on either N1, N2 or N3. In all instances the derived FWHM was larger than the FHWM of the PSF in that particular waveband. The nuclei are therefore resolved. This is not surprising, given that the extended stellar distribution dominates the broad-band light and that star formation over extended regions contributes to the emission line flux. On the other hand, it would have been interesting if any of the nuclei had contained an unresolved component. This could have pinpointed the site of an AGN. An emission line point source could be an unresolved narrow line region or broad line region. A broad-band point source could be emission from the AGN or its surrounding torus, although such sources are generally found only in radio-loud galaxies (Chiaberge, Capetti \& Celotti, 1999; Verdoes Kleijn et al. 2002a). As it stands, the ability to detect 
the presence of an AGN in optical imaging remains extremely difficult in these very dusty ULIRGs.

The HST data yield the following J2000 coordinates for the nuclei: $\left(16 \mathrm{~h} 52 \mathrm{~m} 58.914 \mathrm{~s}, 2^{\circ} 24^{\prime} 3.64^{\prime \prime}\right)$ for $\mathrm{N} 1$; (16h $\left.52 \mathrm{~m} 58.900 \mathrm{~s}, 2^{\circ} 24^{\prime} 3.50^{\prime \prime}\right)$ for $\mathrm{N} 1^{\prime}$; $\left(16 \mathrm{~h} 52 \mathrm{~m} 58.859 \mathrm{~s}, 2^{\circ} 24^{\prime} 1.95^{\prime \prime}\right)$ for $\mathrm{N} 2$; and $\left(16 \mathrm{~h} 52 \mathrm{~m} 58.872 \mathrm{~s}, 2^{\circ} 24^{\prime} 1.60^{\prime \prime}\right)$ for N3. For N1, $\mathrm{N} 2$ and N3 these coordinates were measured from the pipeline-reduced I-band image. For N1', which is not seen in the I-band image, the coordinates were measured from the pipeline-reduced K-band image, after aligning it to the I-band image. In an absolute sense, the listed coordinates are accurate only at the level of $\sim 1^{\prime \prime}$, which is the absolute pointing accuracy of HST. However, the relative positions of the components are very accurate, $\lesssim 0.04^{\prime \prime}$. The morphology of the nuclear structure does depend on wavelength, so there may be additional systematic errors due to spatially variable dust absorption.

NGC 6240 shows two nuclear components in radio continuum emission. Carral, Turner \& Ho (1990) and Eales et al. (1990) list coordinates of the components that are in mutual agreement to within $0.1^{\prime \prime}$. The average of their results is $\left(16 \mathrm{~h} 52 \mathrm{~m} 58.923 \mathrm{~s}, 2^{\circ} 24^{\prime} 4.66^{\prime \prime}\right)$ for the Northern component and $\left(16 \mathrm{~h} 52 \mathrm{~m} 58.886 \mathrm{~s}, 2^{\circ} 24^{\prime} 3.27^{\prime \prime}\right)$ for the Southern component. These coordinates differ at the $\sim 1^{\prime \prime}$ level from the coordinates derived from the HST data, as expected on the basis of the absolute accuracy of the latter. The radio components are separated from each other by $1.48^{\prime \prime}$ along position angle $\mathrm{PA}=21.3^{\circ}$ (measured from North over East). Recent observations with MERLIN at $5 \mathrm{GHz}$ (Beswick et al. 2001) are consistent with this result. The angular resolution of these data is about 0.1 arcsec and the separation between the two brightest components seen in the MERLIN data is $1.52^{\prime \prime}$. As discussed in Section 1, Chandra data have recently shown that NGC 6240 also has a double nucleus in hard X-rays. Komossa et al. (2003) quote the distance between the X-ray nuclei as $1.4^{\prime \prime}$, consistent with the distance between the radio nuclei to within the observational errors. The southern nucleus is the brightest one, both at radio and at X-ray wavelengths. The K-band image in Figure $3 \mathrm{c}$ shows that the same is true at near-IR wavelengths.

It it puzzling that the separation determined from the radio data and the X-ray emission does not agree with the separation of the optical HST components. N1 and N2 are separated by $1.86^{\prime \prime}$ along $\mathrm{PA}=25.0^{\circ}$, while $\mathrm{N} 1$ and $\mathrm{N} 3$ are separated by $2.12^{\prime \prime}$ along $\mathrm{PA}=16.0^{\circ}$. This implies that at least one of the radio/X-ray components does not have an optical counterpart, and conversely, that at most one of the optical components has a radio/X-ray counterpart. The K-band image shows a hint of a component $\mathrm{N}^{\prime}$ to the south of $\mathrm{N} 1$ (Figure 3c) that has high extinction. It could be that that component marks the position of the northern radio/X-ray nucleus, but even that does not fully resolve the discrepancy. $\mathrm{N}^{\prime}$ and $\mathrm{N} 2$ are separated along $\mathrm{PA}=21.6^{\circ}$ by $1.67^{\prime \prime}$, which is still larger than the nuclear separation seen in the radio and X-ray data. Especially for the X-ray nuclei, there is strong (spectral) evidence that they represent two AGNs (Komossa et al. 2003). Since super-massive black holes are generally found in the regions of highest stellar density, this suggests strongly that even in the K-band we may not be seeing all the way through the dust. 


\section{STIS Spectroscopy}

\subsection{Observations and Data Reduction}

Long-slit spectra of NGC 6240 were obtained with STIS on HST between May 1999 and June 2000 in the context of HST program GO-8261 (PI: van der Marel). The STIS instrument is described in detail in Proffitt et al. (2002). A total of ten orbits were available to study the kinematics of the galaxy. Half of the time was used to study the nuclear region. The other half was used to study a location about 12 arcsec east of the nuclear region where a steep gradient in the velocity field of the ionized gas was previously detected in ground-based data. For the nuclear region we obtained both emission-line spectra and absorption line spectra. These spectra totaled 2 and 3 orbits, respectively, and were all obtained in a single visit. For the off-nuclear region we only obtained emission-line spectra. These were obtained in two separate visits of 2 and 3 orbits, respectively. All spectra were obtained using the G750M grating, which has a dispersion of $0.56 \AA$ per pixel. The spatial scale along the slit is $0.05^{\prime \prime}$ per pixel. The emission line spectra cover the spectral range 6484-7054 $\AA$, centered approximately on the redshifted wavelength of $\mathrm{H} \alpha+[\mathrm{NII}]$. The associated velocity scale is $24.8 \mathrm{~km} \mathrm{~s}^{-1}$ per pixel. The absorption line spectra cover the spectral range 8539-9111 $\AA$, centered approximately on the redshifted wavelength of the redshifted Ca II triplet. The associated velocity scale is $19.0 \mathrm{~km} \mathrm{~s}^{-1}$ per pixel.

To position the slits we started each visit with a target acquisition on an uncatalogued star of magnitude $V=20.8$ located $18^{\prime \prime}$ from the nuclear region of NGC 6240. This star has J2000.0 coordinates $\left(16 \mathrm{~h} 52 \mathrm{~m} 59.847 \mathrm{~s}, 2^{\circ} 24^{\prime} 12.60^{\prime \prime}\right)$, to within the $\sim 1^{\prime \prime}$ accuracy of the HST Guide Star Coordinate System. The standard ACQ acquisition procedure was used with a 100 sec exposure time. This yields a nominal positional accuracy of $\lesssim 0.02^{\prime \prime}$ (Proffitt et al. 2002). The telescope was subsequently slewed to the position of interest. The commanded slews were measured from the WFPC2 images to $\lesssim 0.01^{\prime \prime}$ accuracy. The accuracy with which small slews are executed by HST makes a negligible contribution to the overall positional error budget.

The spectra were calibrated by the HST calibration 'pipeline' maintained by STScI. The standard reduction steps include bias subtraction, dark current subtraction, flat-fielding,

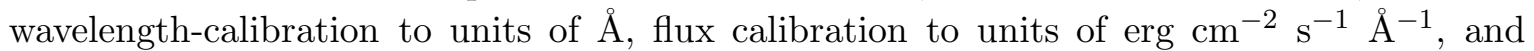
two-dimensional rectification to linear spatial and wavelength scales. At each slit position two or more exposures were obtained. These were dithered by an integer number of pixels in the direction parallel to the slit, to allow removal of CCD defects during data reduction. Different exposures for the same slit position were aligned, and then combined with simultaneous removal of cosmic rays and chip defects. The wavelength calibration was performed using arc-lamp spectra obtained in the same visits as the science spectra. This yields wavelength scales accurate to $0.1-0.2$ spectral pixels, i.e., $\lesssim 5 \mathrm{~km} \mathrm{~s}^{-1}$.

The emission-line spectra were analyzed by simultaneous fitting of Gaussian profiles to the emission lines using the software described in van der Marel \& van den Bosch (1998). The wavelength range covered by the spectra includes five emission lines: $\mathrm{H} \alpha$ at a vacuum rest-wavelength of $6564.6 \AA$, [NII] lines at $6549.8 \AA$ and $6585.3 \AA$, and [SII] lines at $6718.3 \AA$ and $6732.7 \AA$. The $S / N$ of the [SII] lines was generally low and in the following we discuss only the 
$\mathrm{H} \alpha+[\mathrm{NII}]$ lines. The Gaussian fits yield the flux, mean radial velocity, and Gaussian velocity dispersion $(\sigma=\mathrm{FWHM} / 2.355)$ of the lines.

The absorption-line spectra were rebinned onto a logarithmic scale and were then analyzed using software that performs a fit in pixel space (van der Marel 1994). The galaxy spectrum was modeled as the convolution of a template spectrum and a Gaussian line-of-sight velocity profile. The signal-to-noise ratio of the absorption line spectra was insufficient to measure deviations of the velocity profile shapes from a Gaussian, so no attempt was made to measure higher-order Gauss-Hermite moments. The best-fit parameters of the Gaussian velocity profile were determined by minimizing the residuals of the fit in a $\chi^{2}$ sense. This yields the mean stellar velocity, the velocity dispersion, and a line-strength parameter (a measure of the equivalent width of the absorption lines in the galaxy spectrum with respect to those in the template spectrum). The analysis uses all absorption lines in the spectrum, but most of the signal is contained in the three Ca II absorption lines at vacuum rest-wavelengths of $8500.4 \AA, 8544.4 \AA$ and $8664.5 \AA$. Template spectra were not observed as part of our project, so we used a STIS spectrum of the star HR 7615 (type K0III) from the HST Data Archive (project GO-7566, PI: Green).

\subsection{Kinematics of the Nuclear Region}

\subsubsection{Gas Kinematics}

The gas kinematics of the nuclear region was studied by obtaining spectra for seven different slit positions. The slits were aligned parallel to line that connects the nuclei N1 and N2, using a pattern that covers a contiguous area with a width of $1.1^{\prime \prime}$. The lay-out of the slit positions is shown schematically in Figure 4. The central three positions in the pattern were observed with a slit of $0.1^{\prime \prime}$ width (52x0.1). Two exposures of $250 \mathrm{sec}$ were obtained at each slit position, and no on-chip binning was applied. A wider slit of $0.2^{\prime \prime}$ width $(52 \times 0.2)$ was used for the outer four positions. Two exposures of $300 \mathrm{sec}$ were obtained at each slit position, and two-pixel on-chip binning was applied in both the spatial and spectral directions (yielding effective pixels of $0.10^{\prime \prime}$ by $1.12 \AA=49.6 \mathrm{~km} \mathrm{~s}^{-1}$ ). This strategy was adopted to increase the signal-to-noise ratio $(S / N)$ of the spectra in areas where the emission line flux is low. The spectral resolution for an extended source observed with the $0.1^{\prime \prime}$-wide slit is $\sim 21 \mathrm{~km} \mathrm{~s}^{-1}$ (Gaussian dispersion), and for the $0.2^{\prime \prime}$-wide slit it is $\sim 42 \mathrm{~km} \mathrm{~s}^{-1}$.

The spectra were analyzed by fitting Gaussians to the emission lines as described above. In principle, each emission line is described by three free parameters. However, the $S / N$ of the spectra is relatively low and the lines are partially blended. We therefore found that more robust results could be obtained by assuming that all emission lines have the same mean velocity and velocity dispersion. This need not be completely true in reality, but is a reasonable assumption in the present context. We also fixed the ratio of the fluxes in the two [NII] lines to the ratio of their transition probabilities (i.e., 3; see references in Mendoza 1983). The ratio of the $\mathrm{H} \alpha$ and

[NII] fluxes was kept as a free parameter. The best-fitting Gaussian emission-line parameters were determined as a function of position along the slit. For the observations with the $0.1^{\prime \prime}$ slit we applied two-pixel binning along the slit to increase the $S / N$. The final sampling along the slit was 
therefore $0.1^{\prime \prime}$ for both the narrow-slit and the wide-slit observations.

The emission-line fit results for the different slit positions were combined and interpolated to yield two-dimensional maps of the flux, radial velocity and velocity dispersion of the nuclear region of NGC 6240. The results are shown as two-dimensional greyscale and contour maps in Figure 5. To get a different sense of these data, Figure 6 shows the projection along the $Y$-axis of Figure 5 . To obtain the latter results we summed the fluxes obtained for the seven different slit positions and analyzed the results with the same Gaussian-fitting method as described above. Hence, Figure 6 shows the kinematics for a hypothetical 1.1"-wide slit through N1 and N2, as function of position along the slit. Here and throughout this paper we subtracted the systemic velocity $v_{\mathrm{sys}}=7287 \pm 8 \mathrm{~km} \mathrm{~s}^{-1}$ of NGC 6240 (Falco et al. 1999) from the mean velocity measurements.

The flux distribution inferred from the spectra (Figure 5a) corresponds well with the $\mathrm{H} \alpha+[\mathrm{NII}]$ narrow band image (Figure 3b; which is rotated with respect to Figure 5a). The two nuclei are clearly visible. The northern nucleus N1 (left in Figure 5) has the higher emission-line flux. The telescope was commanded to place N2 at the origin of the coordinate system of Figure 5. It is therefore clear that the offset-star target acquisition and telescope slew procedures described in Section 3.1 were accurate to better than $\sim 0.03^{\prime \prime}$, as expected.

The ionized gas velocity field does not show clear signs of rotation around either of the two nuclei, or equilibrium motion in general. Both N1 and N2 appear to be located in regions of lower mean velocity than their surroundings. The velocity of $\mathrm{N} 2$ is $\sim 250 \mathrm{~km} \mathrm{~s}^{-1}$ lower than that of N1. The highest mean velocity is seen between N1 and N2, and at this point the mean velocity is $600 \mathrm{~km} \mathrm{~s}^{-1}$ larger than the mean velocity of N2. A similar velocity gradient between the nuclei was reported from observations of CO gas by Tacconi et al. (1999), and was seen also in HI absorption by Beswick et al. (2001). While Tacconi et al. found the CO velocity field to be highly complex, they were able to fit it with a model of a rotating disk between the two nuclei. The mean velocity profile in Figure $6 \mathrm{~b}$ is not inconsistent with this picture. The profile is approximately antisymmetric around a position between N1 and N2, suggestive of rotation. However, the two-dimensional map in Figure 5b is quite complex and it is unlikely that it can be fit with any simple equilibrium model. For the case of the $\mathrm{CO}$ velocity field, the suggestion of a rotating disk between the nuclei received some support from the fact that the observed CO flux actually peaks between the two nuclei. On the other hand, the ionized gas emission that we observe clearly does not peak between the two nuclei (see Figures 5a and 6a). This makes it hard to explain why the ionized gas and the molecular gas have similar kinematics. More generally, gas disks have often been argued to exist around the nuclei of ULIRGs (Sakamoto et al. 1999), but only rarely between them. Current N-body simulations do not yet have sufficient resolution to follow the gaseous and stellar kinematics inside the central kpc of mergers in much detail. However, they do indicate that the dynamics can be complex and out of equilibrium, and that projection effects from multiple kinematic components can lead to very disorganized kinematics (Mihos 2000). Indeed, such complex velocity fields from overlapping kinematic components have been observed in other ULIRGs (Mihos \& Bothun 1998) and can only be disentangled through two-dimensional mapping of the velocity fields with high spectral resolution. An interpretation of the gas velocity curve of NGC 6240 (Figure 6b) as simply being due to equilibrium rotation in a organized disk may therefore be inappropriate. In view of this, we have refrained from constructing models for 
the velocity curve to attempt a determination of the central mass distribution of the galaxy.

The velocity dispersion of the ionized gas is $\sim 300 \mathrm{~km} \mathrm{~s}^{-1}$ at both $\mathrm{N} 1$ and N2. The velocity dispersion between the nuclei varies, dropping to values near $\sim 200 \mathrm{~km} \mathrm{~s}^{-1}$. Overall, the velocity dispersion shows a complex morphology and is larger than what would be expected for a cold disk — in some cases rising to $>400 \mathrm{~km} \mathrm{~s}^{-1}$. This indicates that there is a strong non-equilibrium or turbulent component to the gas kinematics, not entirely surprising in view of the merging nature of this system. Given the superwind morphology of the ionized gas, the features observed in the velocity maps may arise from outflow kinematics. In a bipolar outflow, one would expect split emission lines (e.g., Heckman et al. 1990); however, at the low $S / N$ of our STIS data we would be unable to detect this splitting, particularly if the outflow cone is filled. In this case, we would only measure an increased velocity dispersion in the gas. Interestingly we also measure the gas to be alternately red- and blue-shifted by about $300 \mathrm{~km} \mathrm{~s}^{-1}$ along the slit when compared to the stellar velocities (see Section 3.2.2 and Figure 7 below), possibly consistent with a bipolar outflow scenario. However, velocity mapping at higher spectral resolution with better two-dimensional coverage than available here is needed to fully address the kinematic state of the ionized gas in NGC 6240.

At the site of the nuclei themselves, we see no dramatic increase in emission line width. Such an increase is often seen in gas kinematical studies of AGNs with HST, even in galaxies that don't have a broad-line region in the traditional sense (e.g., van den Bosch \& van der Marel 1998; Verdoes Kleijn et al. 2000, 2002b). Had this been seen in NGC 6240 as well, it would have provided a clear sign of the AGN(s) responsible for the hard X-rays observed with Chandra. However, even at HST resolution the ionized gas kinematics do not a show a clear black hole signature.

\subsubsection{Stellar Kinematics}

Analysis of stellar kinematics requires higher $\mathrm{S} / \mathrm{N}$ than analysis of gas kinematics, given that absorption lines generally have smaller equivalent widths than emission lines. With the available observing time it was therefore not possible to study the stellar kinematics for a two-dimensional region, as we did for the gas kinematics. Instead, the $0.2^{\prime \prime}$-wide slit was used for only a single slit position. The corresponding spectral resolution for an extended source is $\sim 32 \mathrm{~km} \mathrm{~s}^{-1}$ (Gaussian dispersion). The slit was placed at the center of the pattern shown in Figure 4, thus covering both of the nuclei N1 and N2. Six 1380 sec exposures were obtained, which were co-added during analysis. To reduce the effects of read-out noise, two-pixel on-chip binning was applied in both the spatial and spectral directions (yielding effective pixels of $0.10^{\prime \prime}$ by $1.12 \AA=38.0 \mathrm{~km} \mathrm{~s}^{-1}$ ).

The top panel of Figure 7 shows the continuum intensity as function of position along the slit. The nuclei N1 and N2 are clearly visible. Away from the nuclei, the $S / N$ in the individual rows of the two-dimensional spectrum was rather low. In these regions we co-added spectra along the slit to increase $S / N$. This procedure yielded enough $S / N$ to determine the profile along the slit of the mean stellar velocity, but insufficient $S / N$ to determine the profile of the stellar velocity dispersion. However, it was possible to determine a grand-total average velocity dispersion 
for the central region. Co-addition of all data along the slit with the $x$-coordinate of Figure 7 between $-3^{\prime \prime}$ and $1^{\prime \prime}$ yields $\sigma=200 \pm 40 \mathrm{~km} \mathrm{~s}^{-1}$. These results are in adequate agreement with ground-based measurements of Tecza et al. (2000), who analyzed ground-based observations of the $\mathrm{CO}$ bandhead. They found $\sigma=174 \pm 54 \mathrm{~km} \mathrm{~s}^{-1}$ for N1 and $\sigma=236 \pm 24 \mathrm{~km} \mathrm{~s}^{-1}$ for N2. Tecza et al. (2000) found a hint in their data that the stellar velocity dispersion may be somewhat higher between the two nuclei, $\sigma=276 \pm 51 \mathrm{~km} \mathrm{~s}^{-1}$, but the statistical significance of this result is low.

Both our results and those of Tecza et al. (2000) indicate that the stellar velocity dispersion in the nuclear region is considerably less than the values of $\sim 350 \mathrm{~km} \mathrm{~s}^{-1}$ that were obtained from earlier ground-based observations (Lester \& Gaffney 1994; Doyon et al. 1994). It is possible that these larger values were due to the combined effects of the poorer spatial resolution of these observations and the nuclear gradients in mean velocity reported by Tecza et al. (2000). Lester \& Gaffney (1994) used the perceived high value of $\sigma$ to argue that NGC 6240 is quite massive, while Doyon et al. (1994) used is to argue that NGC 6240 falls roughly on the fundamental plane of elliptical galaxies (supporting the notion that it might be an elliptical galaxy in formation). These arguments are weakened by the lower $\sigma$ values found here with HST and previously by Tecza et al. (2000). More importantly, $\sigma$ may be influenced considerably by the non-equilibrium kinematics of the merger, and it is risky to use it as an equilibrium measure of mass and fundamental-plane position.

To determine the profile of the mean stellar velocity along the slit we performed Gaussian velocity profile fits for which the velocity dispersion was kept fixed to the aforementioned value. Although this ignores possible variations in $\sigma$ along the slit, this does not affect the inferred mean velocities. This is because, to lowest order, the mean and dispersion of a Gaussian are statistically uncorrelated quantities (van der Marel \& Franx 1993). The solid symbols in the bottom panel of Figure 7 shows the inferred mean stellar velocities as function of position along the slit. For comparison, the ionized gas velocities from Figure 6 are overplotted as open symbols. It is clear that the stellar velocities differ considerably from the gas velocities. The stars do not show the large velocity gradient between the nuclei that is seen for both the ionized gas and for the cold CO gas (Tacconi et al. 1999). The mean stellar velocities of N1 and N2 are similar. By contrast, the mean emission line velocity of $\mathrm{N} 1$ is $\sim 250 \mathrm{~km} \mathrm{~s}^{-1}$ lower than for N2. The fact that the stars and gas associated with the nuclei of NGC 6240 do not have the same mean velocities indicates that line-of-sight projection effects, dust absorption, and non-equilibrium merger dynamics may be playing an important role in the observed kinematics of NGC 6240. Obviously, this complicates attempts at interpretation in terms of simple equilibrium models.

The main advantage of our data over the work of Tecza et al. is that our spatial resolution of $\sim 0.2^{\prime \prime}$ is better than their $0.8^{\prime \prime}$ ground-based seeing FWHM. However, our one-dimensional coverage yields only limited insight as compared to the two-dimensional stellar kinematical velocity field that Tecza et al. obtained. The mean stellar velocity profile that we infer between the nuclei is not too dissimilar from the findings of Tecza et al. However, they found in addition that there are considerable stellar velocity gradients of $\sim 350 \mathrm{~km} \mathrm{~s}^{-1}$ peak-to-peak at the positions of both of the nuclei. The kinematic major axis of these gradients do not coincide with the line that connects the nuclei, and as a result, these gradients are not evident in our data. Tecza et al. (2000) interpreted their results as evidence for two rotating stellar disks at the positions of each of the nuclei. While this is certainly a possibility, an alternative view is that these gradients 
may represent non-equilibrium dynamics associated with the galaxy merger (Mihos 2000).

\subsection{Kinematics of the Off-Nuclear Region}

Bland-Hawthorn et al. (1991; hereafter BH91) determined the large scale velocity field of the emission-line gas in NGC 6240. They found a steep gradient in the gas velocity at a position $\sim 11.5^{\prime \prime}$ East and 2.5" North of the nuclear region (white dot in Figure 2). The velocity field there resembles a normal disk rotation curve and they argued that this could be the center of one of the two merging progenitor disks. With the assumption that the gas is in circular rotation, they inferred the presence of $\sim 10^{11} \mathrm{M}_{\odot}$ of material at the position of the velocity gradient. In the absence of a significant amount of starlight at this position, they referred to this mass as a "dark core". They discussed various possible physical interpretations of this result, and suggested a super-massive black hole as the most plausible one.

We used the Royal Greenwich Observatory (RGO) Spectrograph with the $25 \mathrm{~cm}$ camera on the AAT on May 9, 1991 to obtain new ground-based long-slit emission-line spectra of NGC 6240. The R1200 grating was used with a $1.3^{\prime \prime}$ slit, yielding a spectral resolution of $\sim 70 \mathrm{~km} \mathrm{~s}^{-1} \mathrm{FWHM}$. The exposure time was 1800 seconds. The slit was centered on the hypothesized black hole position and was placed along PA $=155$ degrees (indicated by the white line in Figure 2). This is along the direction of the steepest velocity gradient reported in BH91. The spatial resolution of the AAT observations was $\sim 1.5^{\prime \prime}$ FWHM. The inferred $\mathrm{H} \alpha+[\mathrm{NII}]$ velocity curve is shown in Figure 8a. The amplitude of the AAT velocity gradient is similar to that seen in the Fabry-Perot measurements of BH91 but is somewhat smaller than the gradient seen in the Calar Alto observations of the latter authors. This is probably due to differences in spatial resolution among the observations. Either way, the new AAT spectra confirm the existence of a steep velocity gradient that resembles a rotation curve.

Identification of the most massive black holes in the nearby universe is important for understanding both galaxy and black hole assembly over cosmic time. The existence of bright quasars at $z \sim 6$ indicates that $\sim 10^{9} \mathrm{M}_{\odot}$ black holes already existed early in the Universe. Such black holes could have grown considerably through subsequent accretion. However, developments since 1991 have made the presence of a $\sim 10^{11} \mathrm{M}_{\odot}$ black hole at an off-nuclear position in NGC 6240 seem unlikely. First, observations at different wavelengths (optical, near-IR, X-rays) do not show any special feature or source at the suggested black hole position. This is true also for the HST broad-band and narrow-band imaging that we have presented here. Colbert, Wilson \& Bland-Hawthorn (1994) did not detect significant radio emission (but super-massive black holes can of course be radio-quiet). Second, it seems likely that the double nucleus marks the centers of the two progenitor galaxies involved in the merger, in particular now that it is known that both nuclei harbor an AGN (Komossa et al. 2003). Third, $\sim 10^{11} \mathrm{M}_{\odot}$ seems exceedingly massive for a black hole. The most massive black holes known have masses of $\sim 3 \times 10^{9} \mathrm{M}_{\odot}$ (Tremaine et al. 2002). Since black hole mass correlates well with bulge mass and velocity dispersion, there is currently no expectation that galaxies exist with central black holes as massive as $\sim 10^{11} \mathrm{M}_{\odot}$. 
Indepent of its origin, the off-nuclear velocity gradient remains intriguing. We therefore decided to study it further using $\mathrm{H} \alpha+[\mathrm{NII}]$ emission-line spectra with STIS. The emission-line flux in NGC 6240 decreases sharply with distance from the nuclear region (see Figure 2) and it is quite low at the position of the velocity gradient. To accumulate sufficient signal we chose to use the $0.5^{\prime \prime}$-wide slit of STIS (52x0.5). Observations were obtained for seven parallel slit positions, all placed along $\mathrm{PA}=155$ degrees. One of the slits was placed at the position reported by BH91. The other six slit positions were obtained by moving the slit perpendicular to its length (i.e., in the direction of position angle $245^{\circ}$ ) by distances $y=-1.0^{\prime \prime},-0.5^{\prime \prime},+0.5^{\prime \prime},+1.0^{\prime \prime},+1.5^{\prime \prime}$ and $+2.0^{\prime \prime}$, respectively. This yields contiguous coverage of a $3.5^{\prime \prime}$ wide area.

Several individual $380 \mathrm{sec}$ exposures were obtained for each slit position. The number of exposures varied from 5 for $y=-1.0^{\prime \prime}$ (furthest from the nuclear region, where the emission line flux is lowest) to 2 for $y=2.0^{\prime \prime}$ (closest to the nuclear region where the emission line flux is highest). To decrease the effects of read-out noise, four-pixel on-chip binning was applied in both the spatial and spectral directions (yielding effective pixels of $0.202^{\prime \prime}$ by $2.24 \AA=99.2 \mathrm{~km} \mathrm{~s}^{-1}$ ). The effective spatial apertures for the observations are $0.2^{\prime \prime}$ (along to the slit) by $0.5^{\prime \prime}$ (perpendicular to the slit). While the corresponding spatial resolution is admittedly low for HST standards, it is still considerably better than the $\gtrsim 1.5^{\prime \prime}$ resolution data that has been obtained from the ground. The spectral resolution for an extended source observed with the $0.5^{\prime \prime}$-wide slit is $\sim 105 \mathrm{~km} \mathrm{~s}^{-1}$ (Gaussian dispersion). This large value does not cause problems because mean velocities of emission lines can be determined with an accuracy that is smaller than their width.

The analysis of the spectra proceeded similarly as described in Section 3.2.1. It was found that the velocity dispersion of the gas showed little systematic variation over the spatial region under study, and was everywhere consistent with $\sigma \approx 250 \pm 50 \mathrm{~km} \mathrm{~s}^{-1}$. The mean velocity results shown in the figure were obtained by keeping $\sigma$ fixed to this value in the emission line fits. Given the low $S / N$ of the data, it was found that this reduced the noise in the inferred mean velocity profiles. The results for the different slits were combined to form the two-dimensional map of the mean gas velocity shown in Figure 9a. The "checkerboard" appearance of the map is due to the fact that it is built up from measurements for individual $0.2^{\prime \prime} \times 0.5^{\prime \prime}$ "apertures". The errors on the velocity measurements for the individual apertures are in the range $50-100 \mathrm{~km} \mathrm{~s}^{-1}$. These relatively large errors are due to the low $S / N$ of the spectra and are responsible for the apparent "graininess" of Figure 9a. Nonetheless, the data provide useful new insight into the velocity field.

Figure $8 \mathrm{~b}$ shows the projection of the data along the $y$-axis of Figure 9a, with additional 5-pixel binning along the slit. In other words, it shows the kinematics for a hypothetical $3.5^{\prime \prime}$-wide slit with $1.0^{\prime \prime}$ pixels, as function of position along the slit. The projection and binning reduce the errors in the mean velocity measurements, and the steep gradient in the gas velocities is more clearly visible than in Figure 9a. Figure 8b shows the results for a much larger range of $x$-values than Figure 9a, to allow comparison with the ground-based data. The STIS results are in good agreement with our AAT data and with the Fabry-Perot data of BH91. The Calar Alto data of the latter authors shows a somewhat larger velocity amplitude, possibly due to a difference in the effective spatial resolution. Either way, the datasets are all in adequate qualitative agreement.

To provide further insight we compared the data to the expected velocities for a model gas 
disk in rotation around black hole. The calculations were performed using the software described in van der Marel \& van den Bosch (1998). It was assumed that the circular velocity of the disk is dominated by the gravity of the black hole, with negligible contributions from stellar gravity. This is motivated by the fact that no concentration of stars is observed at the center of the observed velocity gradient. The calculations take into account the distance and inclination of the gas disk, the STIS PSF, and the binning of the data into individual apertures. The models yield a predicted line profile for each aperture, to which a Gaussian is fitted for comparison to the actual data. For illustrative purposes, we adopt an inclination of $i=60^{\circ}$ for this model disk.

Models of rotation around a black hole always predict that the observed velocity gradient increases as the spatial resolution is improved. For the mass suggested by BH91, the models for the STIS observational setup predict a major axis velocity difference of $1313 \mathrm{~km} \mathrm{~s}^{-1}$ between points at $\Delta x= \pm 0.2^{\prime \prime}$ from the black hole position. However, no gradient of such magnitude is seen in our observed velocity field. The largest gradient in Figure 9 a occurs at $(x, y) \approx\left(0.1^{\prime \prime}, 0.5^{\prime \prime}\right)$ and is only $\sim 490 \mathrm{~km} \mathrm{~s}^{-1}$ between points separated by $\Delta x=0.4^{\prime \prime}$. Hence, we do not see the increase in velocity gradient with improved spatial resolution that would be expected if a black hole were present.

The black hole mass that produces roughly the same velocity gradient as observed is only $6 \times 10^{9} \mathrm{M}_{\odot}$. The velocity field predicted by this model is shown in Figure $9 \mathrm{~b}$. This model provides a reasonable fit to the data along the major axis. However, away from the major axis the fit is not very good. A generic prediction of rotating gas disk models is that the velocity gradient falls off steeply with distance from the major axis. This is clearly seen in Figure 9b. However, in the data the velocity gradient is rather similar for all of the parallel slit positions along which data were taken. This is illustrated further by Figure 9c,d, which are similar to Figure 9a,b, but have an additional Gaussian smoothing with $0.5^{\prime \prime}$ dispersion applied to reduce the noise in the data. Especially at values of $x>0$, the isovelocity contours in the data appear more nearly vertical than they do in the model predictions.

In summary, our HST observations do not support the hypothesis that the off-nuclear velocity gradient in NGC 6240 is due to a black hole, in agreement with other lines of evidence. Instead, it appears to be more plausible to assume that the observed gradient is the combined result of projection effects and non-equilibrium merger kinematics. Mihos (2000) showed that gradients like the one observed in NGC 6240 are observed in N-body simulations of mergers, due to projection effects of multiple, distinct kinematic components. Also, Figure 2 shows that the position of the velocity gradient lies across an ionized filament. So it is possible that the kinematics are strongly influenced by a wind. Alternatively, the kinematics could be affected by projection of the filament on top of background or foreground emission, much like the situation of the projected $\mathrm{H} \alpha$ plume identified in the ULIRG IRAS19254-7245 (the Superantennae; Mihos \& Bothun 1998). 


\section{Conclusions}

We have presented the results of an HST study of the ULIRG NGC 6240. WFPC2 imaging was obtained in the broad B, V, and I-bands, as well as in $\mathrm{H} \alpha+[\mathrm{NII}]$ emission. A NICMOS K-band image from the HST Data Archive was analyzed for comparison. STIS Ca triplet absorption-line spectroscopy was obtained of the nuclear region, and $\mathrm{H} \alpha+[\mathrm{NII}]$ emission line spectroscopy was obtained of both the nuclear region and an off-nuclear position where there is a steep velocity gradient.

The global morphology in the broad-band data shows a complex morphology, consistent with the assumed merger nature of NGC 6240. The emission-line gas shows a spectacular filamentary nebula. This nebula appears consistent with a bipolar superwind, but other interpretations can certainly not be ruled out on the basis of the data presented here. Previous ground-based imaging had shown that NGC 6240 has a double nucleus. However, the nuclear morphology seen with HST is considerably more complicated than what was inferred from previous data. Optical images in both continuum light and emission lines show three nuclear components, which we denote N1, N2, and N3. The nucleus N1 corresponds to the northern nucleus seen in ground-based images; the nuclei N2 and N3 jointly correspond to the southern nucleus seen in ground-based images. The nuclei are spatially resolved in the images. An unresolved component could have pinpointed the site of an AGN, but such an unresolved component is not seen. The southernmost nucleus N3 becomes less prominent in the HST data as one progresses to redder wavelengths. This explains why Schulz et al. (1993) found that the distance between the two nuclei seen in ground-based data decreases with increasing wavelength. From our HST data we conclude that N3 is not a true nucleus, but more likely an HII region or an artifact of patchy dust obscuration. We estimate the absorption $A_{V}$ towards the nuclei with a variety of simple assumptions and obtain values that are broadly consistent with the estimates obtained previously by other authors. The distance between $\mathrm{N} 1$ and N2 is larger than the distance between the radio and X-ray nuclei of NGC 6240. In the $\mathrm{K}$-band an additional component N1' becomes evident close to N1. However, also the distance between $\mathrm{N1}^{\prime}$ and $\mathrm{N} 2$ is larger than the distance between the radio and X-ray nuclei of NGC 6240 . This implies that at least one of the radio/X-ray components does not have an optical/near-IR counterpart, and conversely, that at most one of the optical/near-IR counterpart components has a radio/X-ray counterpart. In other words, even in K-band we may not be seeing all the way through the dust.

The ionized gas velocity field in the nuclear region does not show clear signs of rotation around either of the nuclei, or of equilibrium motion in general. Both N1 and N2 appear to be located in regions of lower mean velocity than their surroundings. The velocity of N2 is $\sim 250 \mathrm{~km} \mathrm{~s}^{-1}$ lower than that of N1. The highest mean velocity is seen between N1 and N2, and at this point the mean velocity is $600 \mathrm{~km} \mathrm{~s}^{-1}$ larger than the mean velocity of N2. A similar velocity gradient between the nuclei was reported from observations of CO gas by Tacconi et al. (1999). The velocity dispersion of the ionized gas is $\sim 300 \mathrm{~km} \mathrm{~s}^{-1}$ at both N1 and N2. The velocity dispersion between the nuclei is lower and drops to values near $\sim 200 \mathrm{~km} \mathrm{~s}^{-1}$, larger than expected for a cold disk. Also, the velocity dispersion map shows a complex morphology. In gas kinematical studies of AGNs with HST one often sees a very sharp increase in emission line width towards the AGN. Although NGC 
6240 harbors two AGNs (Komossa et al. 2003), no dramatic increase in emission line width is seen towards either of the optical nuclei of NGC 6240.

The stellar kinematics in the nuclear region are quite different from the gas kinematics. The stars do not show the large velocity gradient between the nuclei that is seen for both the ionized gas and for the cold CO gas. Also, the mean stellar velocities of N1 and N2 are similar, while the mean emission line velocity of $\mathrm{N} 1$ is $\sim 250 \mathrm{~km} \mathrm{~s}^{-1}$ lower than for N2. We cannot accurately measure the stellar velocity dispersion in the nuclear region from the STIS absorption line spectra. However, our low $S / N$ measurement $\sigma=200 \pm 40 \mathrm{~km} \mathrm{~s}^{-1}$ is not inconsistent with the ground-based results of Tecza et al. (2000). Both measurements indicate that the stellar velocity dispersion in the nuclear region is lower than the values of $\sim 350 \mathrm{~km} \mathrm{~s}^{-1}$ that were obtained from earlier ground-based observations (Lester \& Gaffney 1994; Doyon et al. 1994), probably due to differences in spatial resolution.

We have also studied the emission-line velocity field at an off-nuclear position where Bland-Hawthorn et al. (1991) reported a steep velocity gradient. Both our ground-based AAT spectra and HST/STIS spectra confirm the existence of the gradient. The STIS data do no show a steepening of the gradient compared to the ground-based data. Combined with detailed models and the lack of observed counterpart at other wavelengths this suggests strongly that the velocity gradient is not due to rotation around a black hole.

Overall, the spectroscopic data of NGC 6240 indicate that line-of-sight projection effects, dust absorption, non-equilibrium merger dynamics and the possible influence of a wind may be playing an important role in the observed kinematics. In view of this, it is unclear what the validity is of simple equilibrium models for the observed kinematics (such as those advocated by, e.g., Tacconi et al. 1999 and Tecza et al. 2000), and whether the stellar velocity dispersion can be used as an equilibrium measure of mass or fundamental plane position (as done by Lester \& Gaffney 1994; Doyon et al. 1994).

We would like to thank Zolt Levay for assistance with Figure 2 and David Zurek for assistance in the preliminary reductions of the WFPC2 data. Support for proposals \#6430 and \#8261 was provided by NASA through a grant from the Space Telescope Science Institute, which is operated by the Association of Universities for Research in Astronomy, Inc., under NASA contract NAS 5-26555. JCM acknowledges support by the NSF through grant AST-9876143 and by a Research Corporation Cottrell Scholarship. This research has made use of the NASA/IPAC Extragalactic Database (NED) which is operated by the Jet Propulsion Laboratory, California Institute of Technology, under contract with the National Aeronautics and Space Administration. 


\section{REFERENCES}

Barbieri, C., et al. 1993, A\&A, 273, 1

Barger, A. J., Cowie, L. L., Mushotzky, R. F., \& Richards, E. A. 2001, AJ, 121, 662

Beswick, R. J., Pedlar, A., Mundell, C. G., \& Gallimore, J. F. 2001, MNRAS, 325, 151

Biretta, J., et al. 2001, WFPC2 Instrument Handbook, Version 6.0 (Baltimore: STScI)

Bland-Hawthorn, J., Wilson, A. S., \& Tully, R. B. 1991, ApJ, 371, L19

Carollo, C. M., Franx, M., Illingworth, G. D., \& Forbes, D. A. 1997, ApJ, 481, 710

Carral, P., Turner, J. L., \& Ho, P. T. P. 1990, ApJ, 362, 434

Chiaberge, M., Capetti, A., \& Celotti, A. 1999, A\&A, 349, 77

Colbert, E. J. M., Wilson, A. S., \& Bland-Hawthorn, J. 1994, ApJ, 436, 89

de Jong, R. S. 1996, A\&A, 313, 377

Doyon, R., Wells, M., Wright, G. S., Joseph, R. D., Nadeau, D., \& James, P. A. 1994, ApJ, 437, L23

Eales, S. A., Becklin, E. E., Hodapp, K.-W., Simons, D. A., \& Wynn-Williams, C. G. 1990, ApJ, 365,478

Falco, E., et al. 1999, PASP, 111, 438

Genzel, R., et al. 1998, ApJ, 498, 579

Gerssen, J., van der Marel, R. P., Axon, D. J., Mihos, C., Hernquist, L., \& Barnes, J. E. 2001, in The Central Kiloparsec of Starbursts and AGN: The La Palma Connection, ASP Conference Proceedings Vol. 249. J. H. Knapen, J. E. Beckman, I. Shlosman, \& T. J. Mahoney, eds., p. 665 (San Francisco: Astronomical Society of the Pacific)

Heckman, T. M., Armus, L., \& Miley, G. K. 1987, AJ, 93, 276

Heckman, T. M., Armus, L., \& Miley, G. K. 1990, ApJS, 74, 833

Holtzman, J. A. et al. 1995a, PASP, 107, 156

Holtzman, J. A, Burrows, C. J., Casertano, S., Hester, J. J., Trauger, J. T., Watson, A. M., \& Worthey, G. 1995b, PASP, 107, 1065

Iwasawa, K., \& Comastri, A. 1998, MNRAS, 297, 1219

Keel, W. C. 1990, AJ, 100, 356

Komossa, S., Burwitz, V., Hasinger, G., Predehl, P., Kaastra, J. S., \& Ikebe, Y. 2003, ApJ, 582, L15

Kormendy, J., \& Gebhardt, K. 2001, in 'Proc. 20th Texas Symposium on relativistic astrophysics', AIP conference proceedings, Vol. 586., eds., J. C. Wheeler, \& H. Martel, p. 363 (NY: American Institute of Physics)

Kormendy, J., \& Sanders, D. B. 1992, ApJ, 390, L53

Krist, J., \& Hook, R. 2001, The Tiny Tim User's Guide, (Baltimore: STScI)

Leitherer, C., et al. 1999, ApJS, 123, 3 
Lester, D. F., \& Gaffney, N. I. 1994, ApJ, 431, L13

Lira, P., Ward, M. J., Zezas, A., \& Murray, S. S. 2002, MNRAS, 333, 709

Mendoza, C. 1983, Planetary Nebualae (IAU Symposium No. 103) ed. D.R. Flower (Dordrecht:Reidel), 143

Mihos, C. 2000, in 'Dynamics of Galaxies: from the Early Universe to the Present', Combes, F., Mamon, G. A., \& Charmandaris, V., eds., ASP Conference Series, Vol. 197 (San Francisco: Astronomical Society of the Pacific), 275

Mihos, J. C. \& Bothun, G. D. 1998, ApJ, 500, 619

Pasquali, A., de Grijs, R., \& Gallagher, J. S., 2003, MNRAS, accepted

Persson, S. E., Frogel, J. A., \& Aaronson, M. 1979, ApJS, 39, 61

Proffitt, C., et al. 2002, STIS Instrument Handbook, Version 6.0, (Baltimore: STScI)

Rieke, G. H., Cutri, R. M., Black, J. H., Kailey, W. F., McAlary, C. W., Lebofsky, M. J., \& Elston, R. 1985, ApJ, 290, 116

Rieke, G. H., \& Lebofsky, M. J. 1985, ApJ, 288, 618

Sakamoto, K., Scoville, N. Z., Yun, M. S., Crosas, M., Genzel, R., \& Tacconi, L. J. 1999, ApJ, 514,68

Sanders, D. B., \& Mirabel, I. F. 1996, ARA\&A, 34, 749

Sanders, D. B., Soifer, B. T., Elias, J. H., Madore, B. F., Matthews, K., Neugebauer, G., \& Scoville, N. Z. 1988, ApJ, 325, 74

Schneider, G. 2002, Domains of Observability in the near-infrared with HST/NICMOS and (adaptive optics augmented) large ground-based telescopes (http://nicmosis.as.arizona.edu:8000/REPORTS/NICMOS_AO_WHITEPAPER.html)

Schulz, H., Fried, J. W., Röser, S., \& Keel, W. C. 1993, A\&A, 277, 416

Scoville, N. Z., et al. 2000, AJ, 119, 991

Smail, I., Ivison, R. J., \& Blain, A. W. 1997, ApJ, 490, L5

Soifer, B. T., Neugebauer, G., \& Houck, J. R. 1987, ARA\&A, 25, 187

Tacconi, L. J., Genzel, R., Tecza, M., Gallimore, J. F., Downes, D., \& Scoville, N. Z. 1999, ApJ, 524,732

Tecza, M., Genzel, R., Tacconi, L. J., Anders, S., Tacconi-Garman, L. E., \& Thatte, N. 2000, ApJ, 537,178

Tremaine, S., et al. 2002, ApJ, 574, 740

van der Marel, R. P. 1994, MNRAS, 270, 271

van der Marel, R. P., \& Franx, M. 1993, ApJ, 407, 525

van der Marel, R. P., \& van den Bosch, F. C. 1998, AJ, 116, 2220

van der Werf, P. P., Genzel, R., Krabbe, A., Blietz, M., Lutz, D., Drapatz, S., Ward, M. J., \& Forbes, D. A. 1993, ApJ, 405, 522 
van der Werf, P. P. 2001, in Starburst Galaxies: Near and Far, L. Tacconi, D. Lutz, eds., 151 (Heidelberg: Springer-Verlag)

Verdoes Kleijn, G. A., van der Marel, R. P., Carollo, C. M., \& de Zeeuw P.T. 2000, AJ, 120, 1221

Verdoes Kleijn, G. A., Baum, S. A., de Zeeuw, P. T., \& O’Dea, C. P. 2002a, AJ, 123, 1334

Verdoes Kleijn, G. A., van der Marel, R. P., de Zeeuw, P. T., Noel-Storr, J., \& Baum, S. A. 2002b, AJ, 124, 2524

Vignati, P., et al. 1999, A\&A, 349, L57 


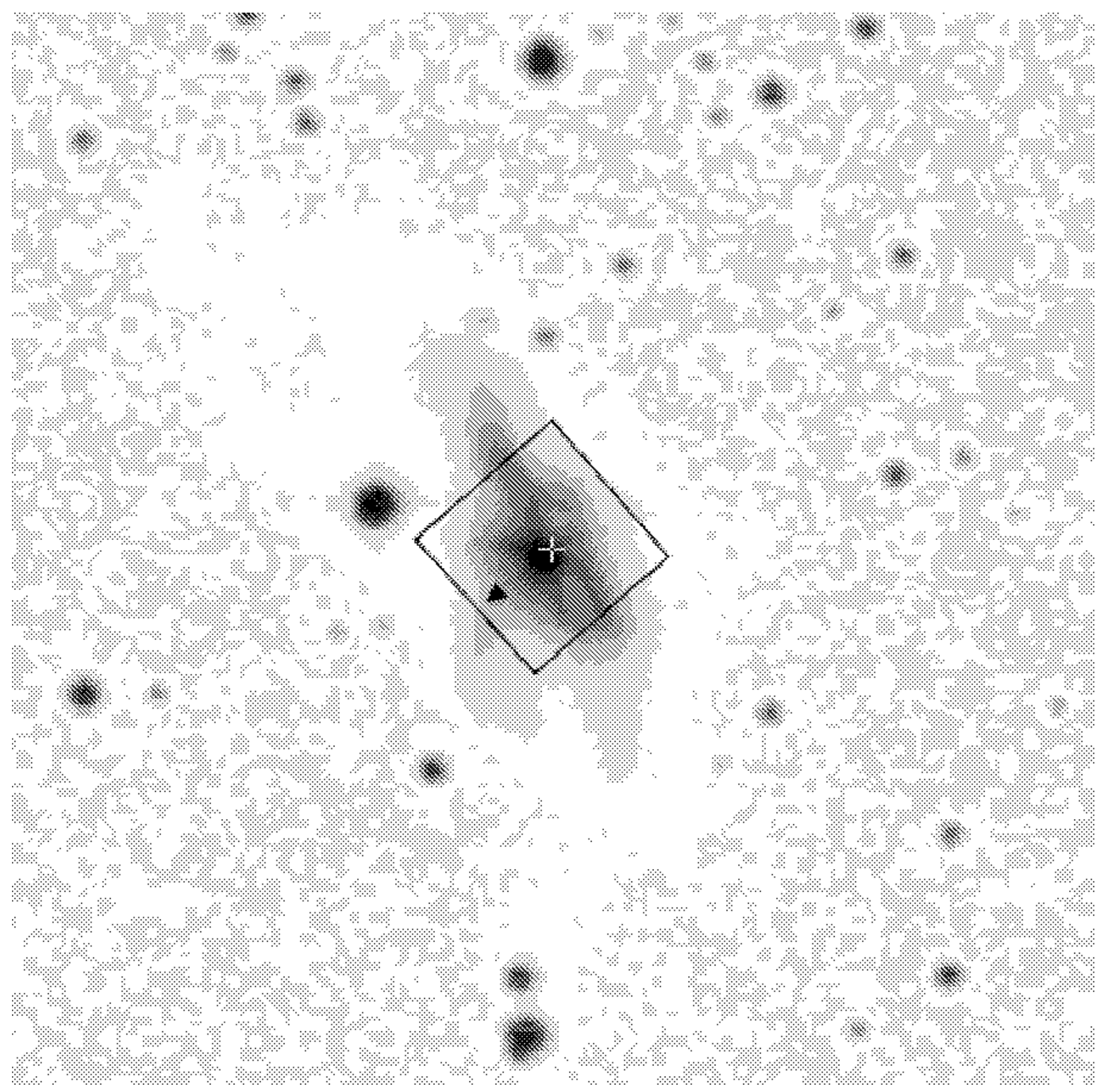

Fig. 1.- Image of NGC 6240 extracted from the Digitized Sky Survey. North is to the top and East is to the left. The cross marks the position of the nuclear region. The square shows the size and orientation of the WFPC2/PC chip field of view $\left(36^{\prime \prime} \times 36^{\prime \prime}\right)$. Figures 2 and 3 are oriented such that the black arrow points towards the bottom of the page. 


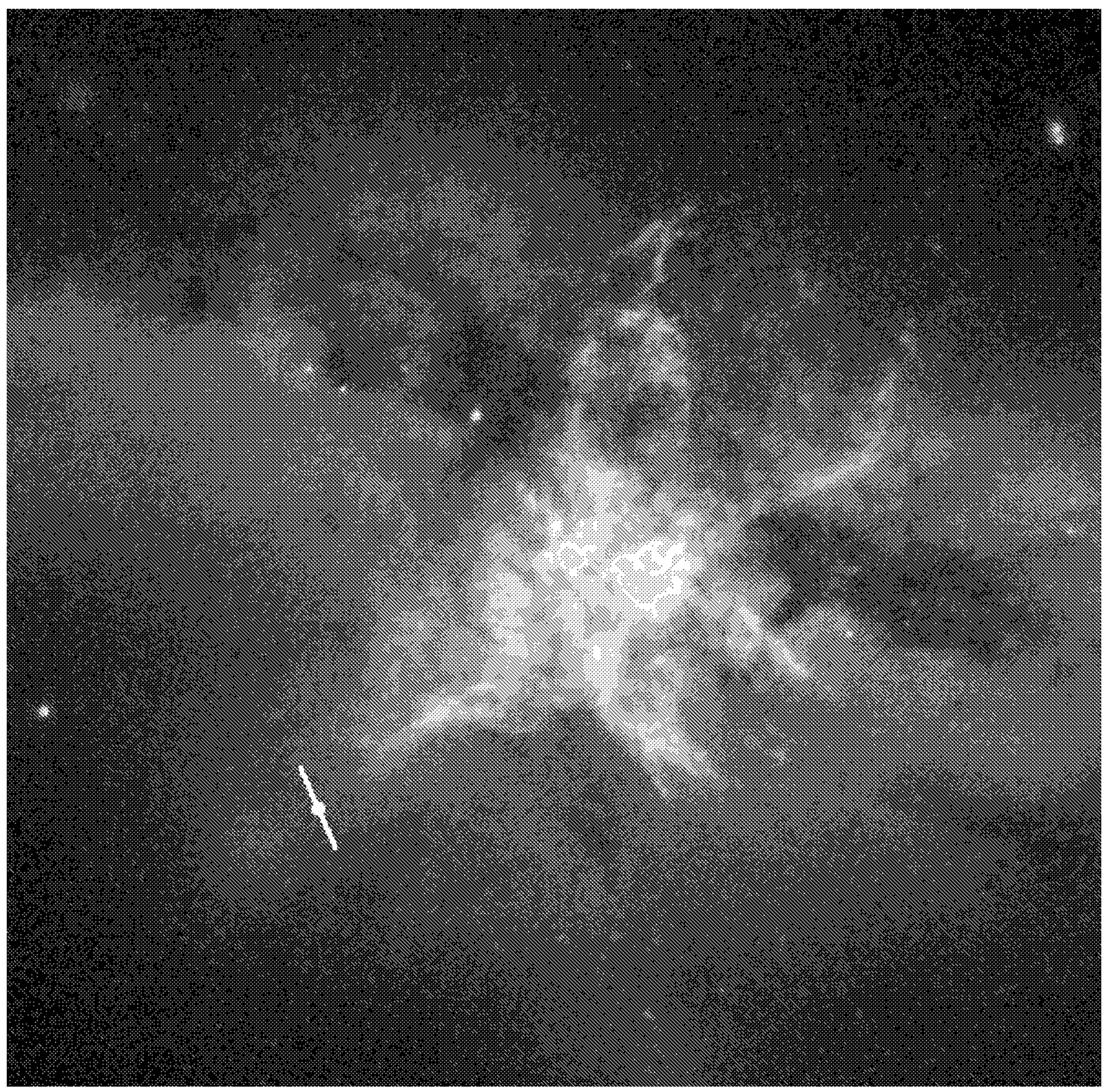

Fig. 2.- Composite WFPC2/PC image of NGC 6240. The field of view and orientation of the image are as indicated in Figure 1; North is at the top left corner of the image. The H $\alpha+[\mathrm{NII}]$ emission image is overlaid in yellow on a true-color image of the continuum light. The latter was constructed from the broad-band $B, V$ and $I$ images. The white dot markes the position at which there is a steep gradient in the emission-line velocities along a position angle of 155 degrees (indicated by the white line). The kinematics in this region are discussed in detail in Section 3.3. 

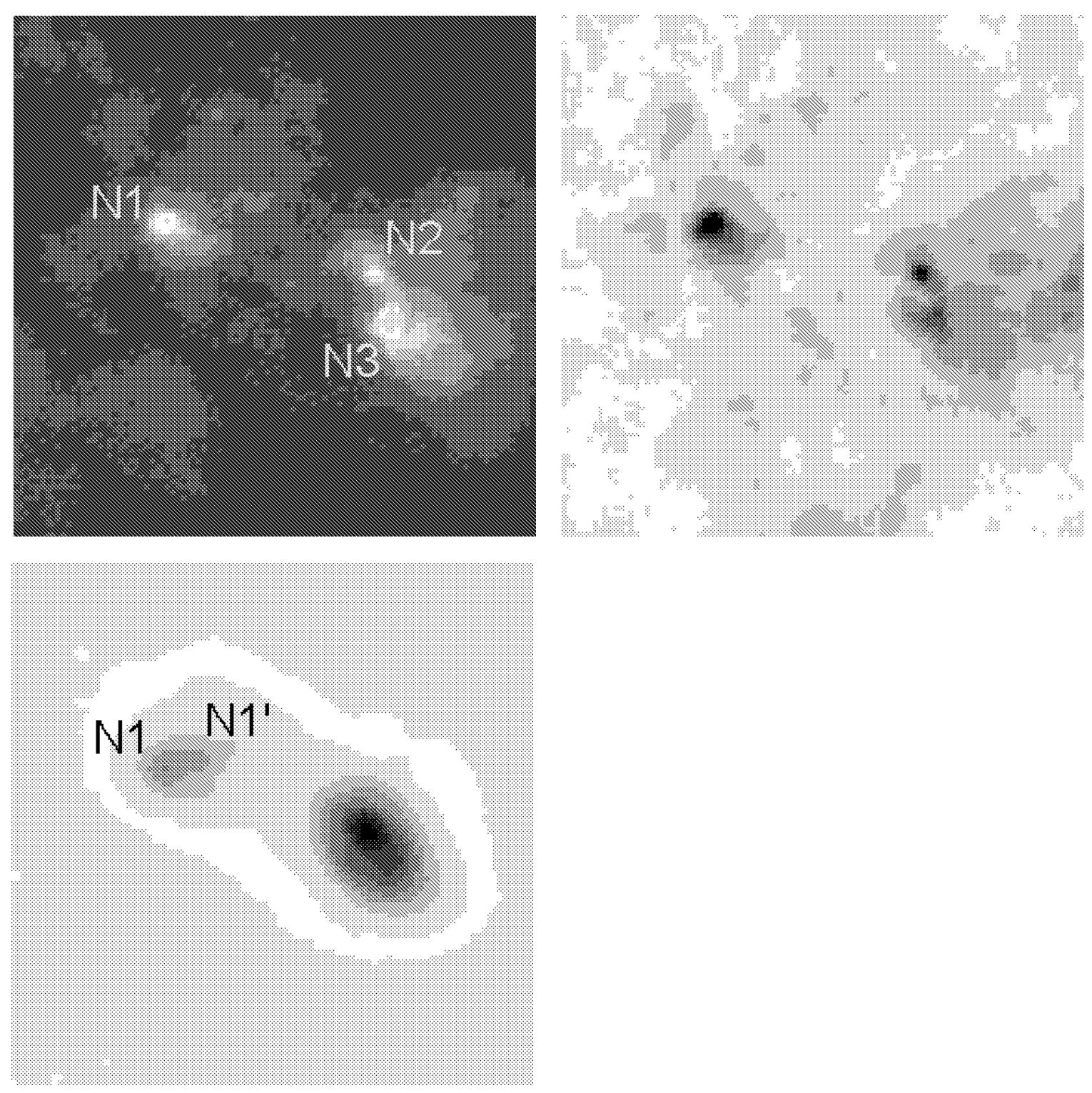

Fig. 3.- The nuclear region of NGC 6240. The image size for each panel is $4.55^{\prime \prime} \times 4.55^{\prime \prime}$. The orientation is the same as in Figure 2 and is as indicated in Figure 1; North is at the top left corner of each panel. (a) True-color image constructed from the WFPC2 broad-band $B, V$ and $I$ images. (b) WFPC2 $\mathrm{H} \alpha+[\mathrm{NII}]$ emission image. (c) NICMOS $K$-band image. The smoother appearance of the $K$-band image compared to the other two is due both to the wider PSF at these wavelengths and the smaller importance of dust absorption. 


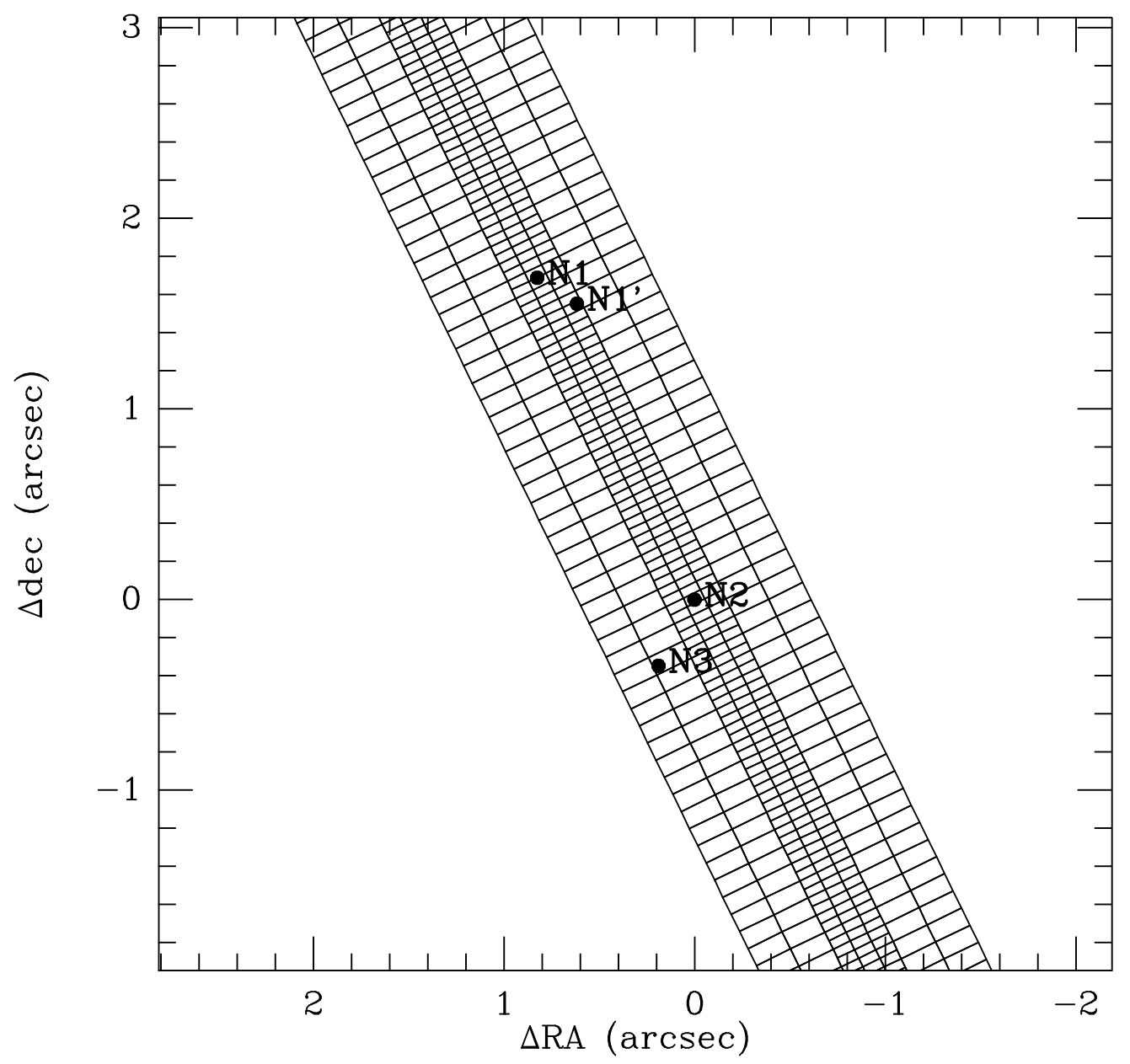

Fig. 4. - Schematic representation of the slit layout for the STIS emission-line spectroscopy of the nuclear region. Spectra were obtained for seven slit positions, as shown in the figure and discussed in the text. The central slits have widths of $0.1^{\prime \prime}$, the outer slits have widths of $0.2^{\prime \prime}$. The pixels along the slits are indicated. Two-pixel on-chip binning was used for the $0.2^{\prime \prime}$-wide slit observations while no on-chip binning was used for the $0.1^{\prime \prime}$-wide slit observations. The nuclear components N1, N1', N2 and N3 of NGC 6240 are as indicated in Figure 3. North is up in this figure, by contrast to Figures 2 and 3. The origin of the coordinate system was arbitrarily chosen at N2. 

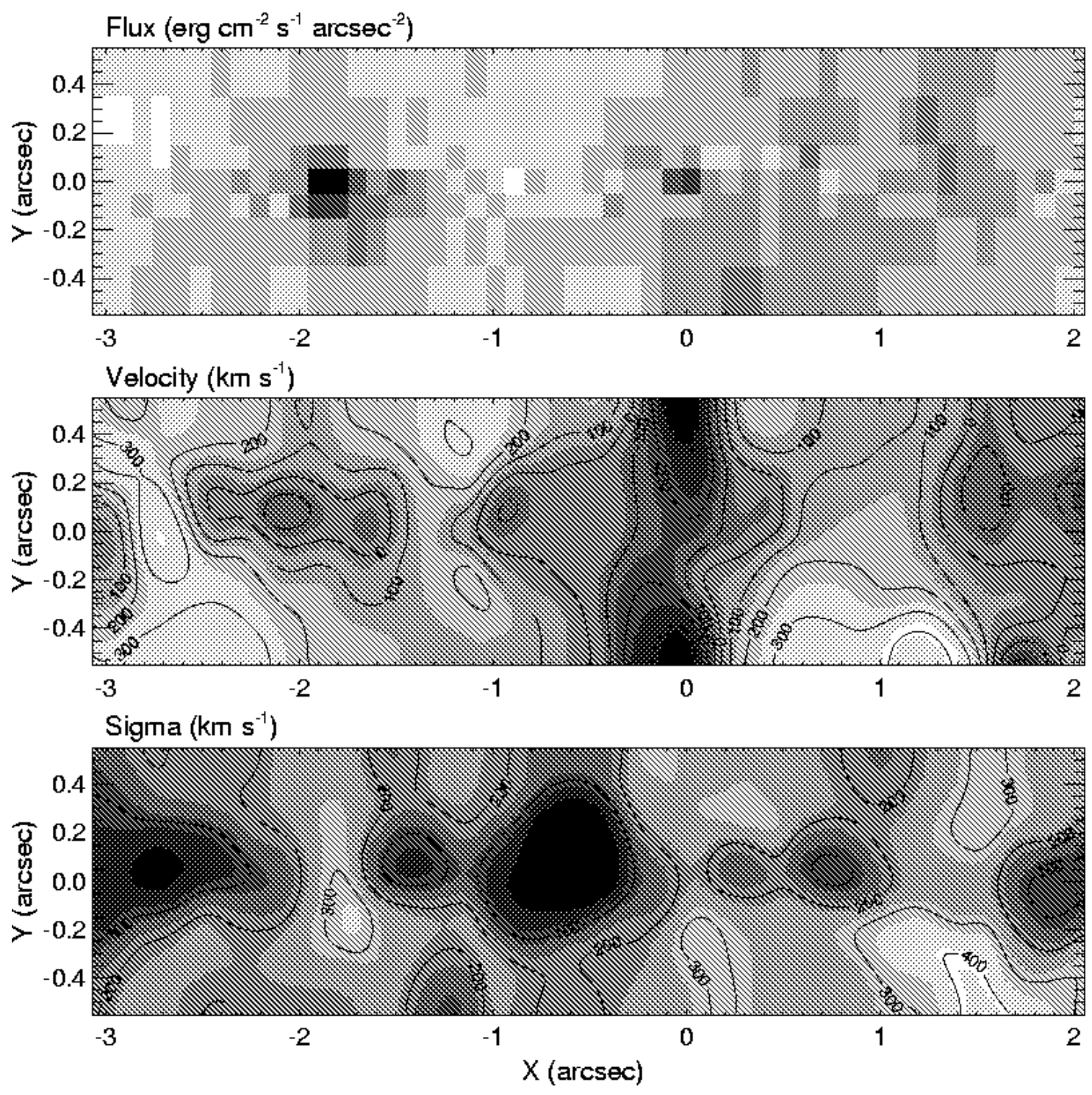

Fig. 5.- Two-dimensional grayscales maps of the $\mathrm{H} \alpha+[\mathrm{NII}]$ emission line properties in the nuclear region of NGC 6240, derived from HST/STIS spectroscopy: (a; top) Total flux; (b; middle) mean velocity; (c; bottom) velocity dispersion. The Cartesian $(X, Y)$ coordinate system is centered on $\mathrm{N} 2$, and has the $X$-axis along the line that connects $\mathrm{N} 1$ at $(X, Y)=\left(-1.86^{\prime \prime}, 0.0^{\prime \prime}\right)$ to N2. The flux distribution overall agrees well with that seen in the $\mathrm{H} \alpha+[\mathrm{NII}]$ WFPC2 narrow band image (Figure 3b, which is rotated with respect to the present figure). The nuclei are clearly visible as peaks in the flux distribution. The pixelized appearance of the flux map is due to the fact that the nuclear region of NGC 6240 was mapped spectroscopically with a set of disjunct "apertures", as shown in Figure 4. By contrast, the mean velocity and velocity dispersion maps were smoothed with a Gaussian of $0.1^{\prime \prime}$ dispersion, to increase $S / N$. These maps have contours overlaid which are labeled in $\mathrm{km} / \mathrm{s}$. 

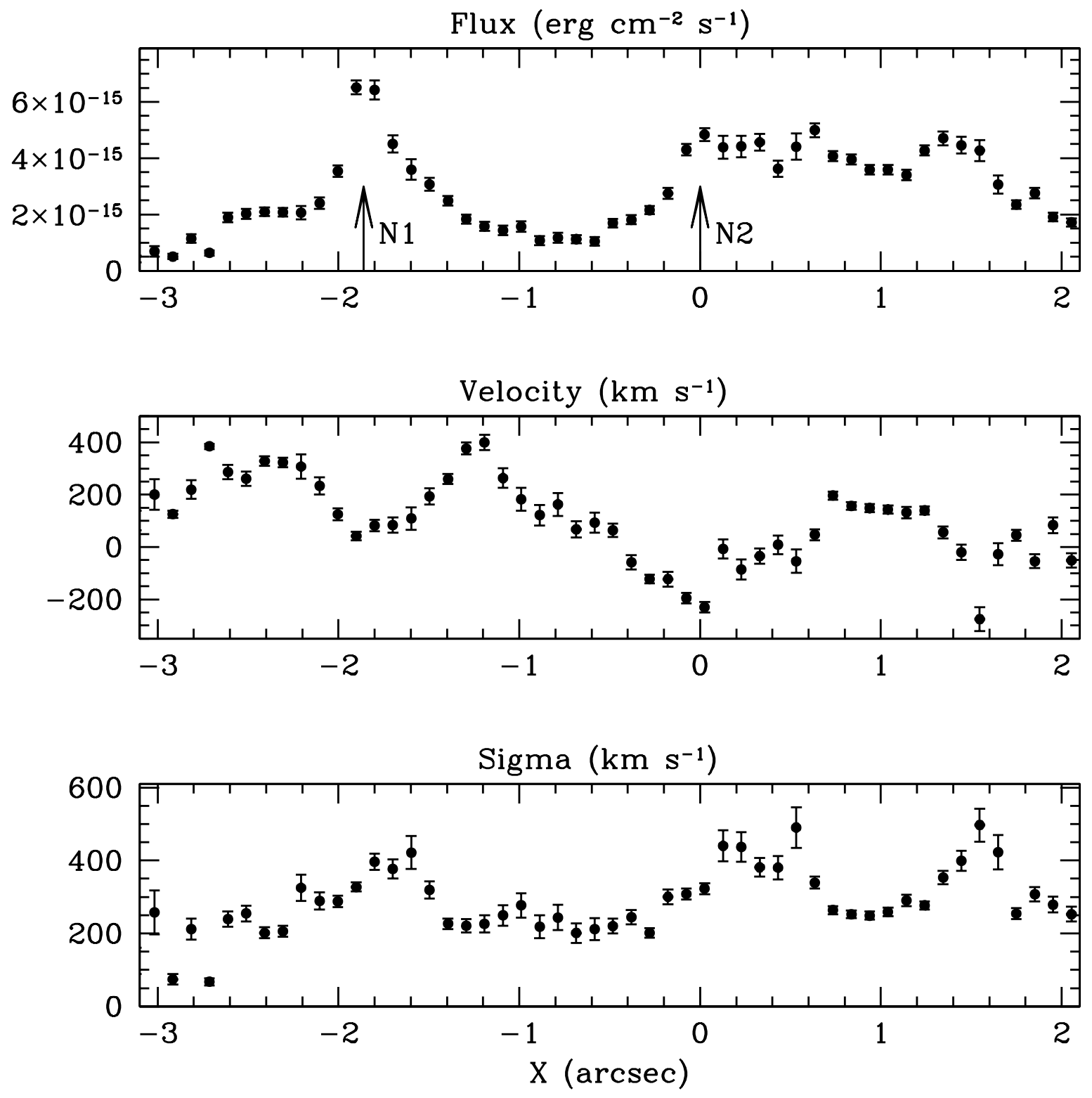

Fig. 6.- $\mathrm{H} \alpha+[\mathrm{NII}]$ emission line properties along a hypothetical $1.1^{\prime \prime}$-wide slit through $\mathrm{N} 1$ and $\mathrm{N} 2$, as function of position along the slit. (a; top) Total flux (in an area of $0.1^{\prime \prime}$ along the slit by $1.1^{\prime \prime}$ perpendicular to the slit); (b; middle) mean velocity; (c; bottom) velocity dispersion. Arrows indicate the positions of the nuclei N1 and N2. These results were obtained as described in the text, by co-adding the data for the individual slit positions shown in Figure 4. This essentially yields a projection of the contour plots in Figure 5 along the $Y$-axis of that figure. 

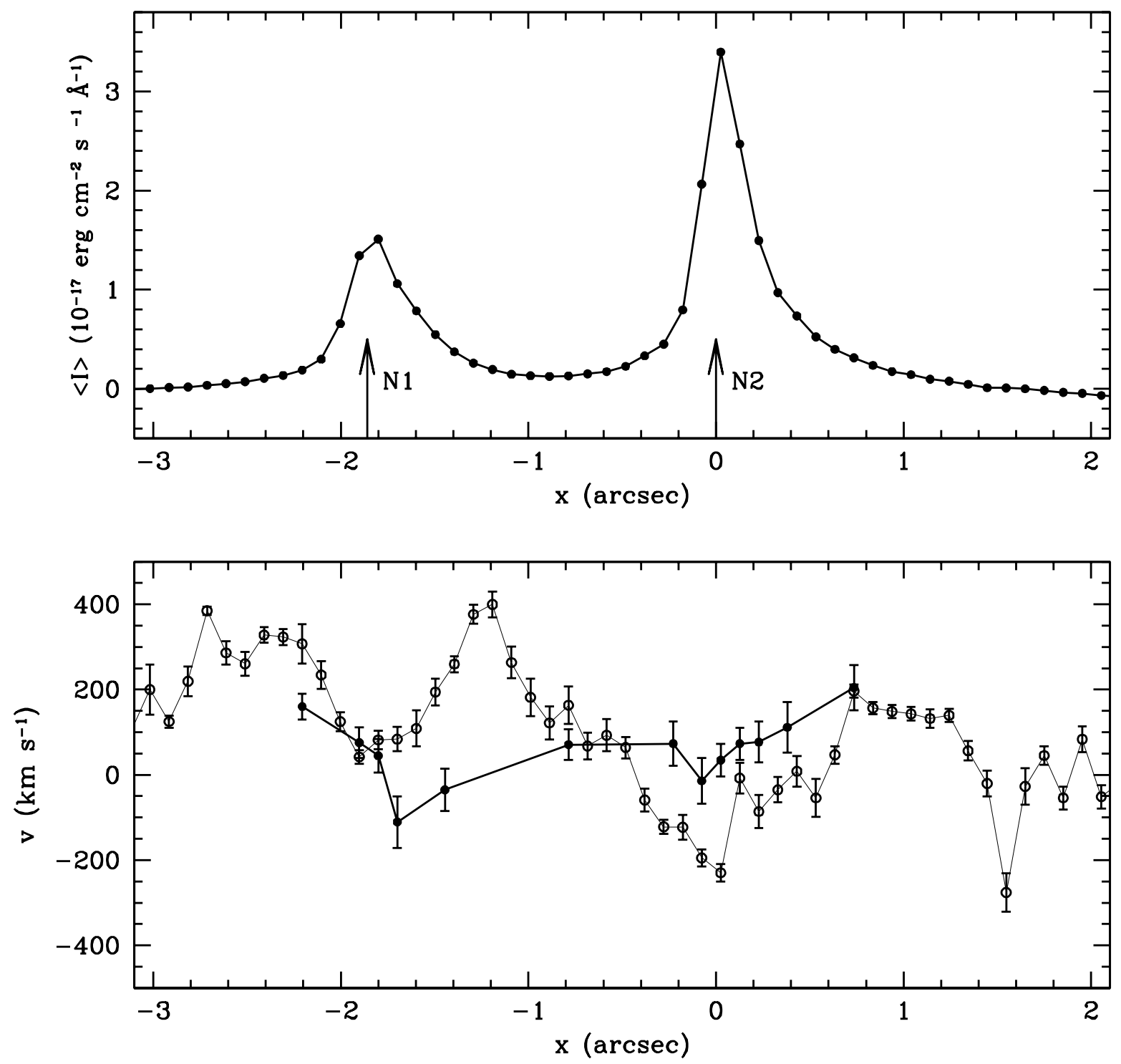

Fig. 7.- Results of Ca II triplet absorption line spectroscopy with a $0.2^{\prime \prime}$-wide slit along the position angle that connects $\mathrm{N} 1$ and N2, as function of position along the slit. (a; top) Average continuum intensity over the observed wavelength range (in an area of $0.1^{\prime \prime}$ along the slit by $0.2^{\prime \prime}$ perpendicular to the slit). The positions of N1 and N2 are indicated. (b; bottom) Comparison of inferred mean stellar velocities (solid symbols) to the mean emission line velocities copied from Figure 6 (open symbols). The stellar velocities differ considerably from the gas velocities. 

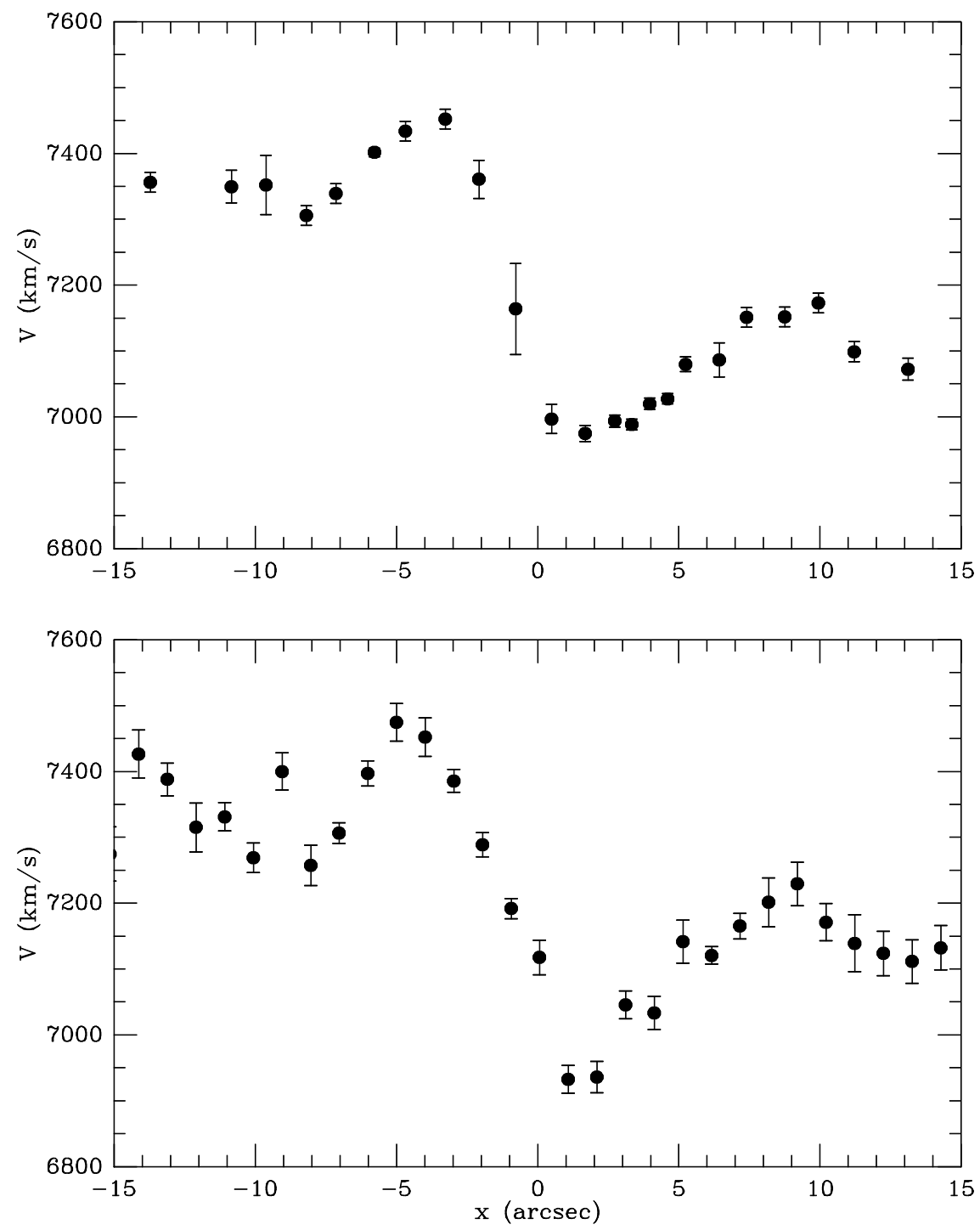

Fig. 8.- Velocity curve of $\mathrm{H} \alpha+[\mathrm{NII}]$ emission line gas at the off-nuclear position indicated in Figure 2. Positive values of the abscissa $x$ lie in the direction of $\mathrm{PA}=155$ degrees. (a; top panel) ground-based AAT data. (b; bottom panel) The HST/STIS results of Figure 9 projected along the $y$ direction with 5-pixel binning along the $x$ direction (corresponding to a hypothetical $3.5^{\prime \prime}$ wide slit with $1.0^{\prime \prime}$ pixels). The results in the two panels are in adequate agreement and confirm the steep velocity gradient reported by Bland-Hawthorn et al. (1991; note that their figure 2 has the horizontal axis flipped compared to the present figure). 

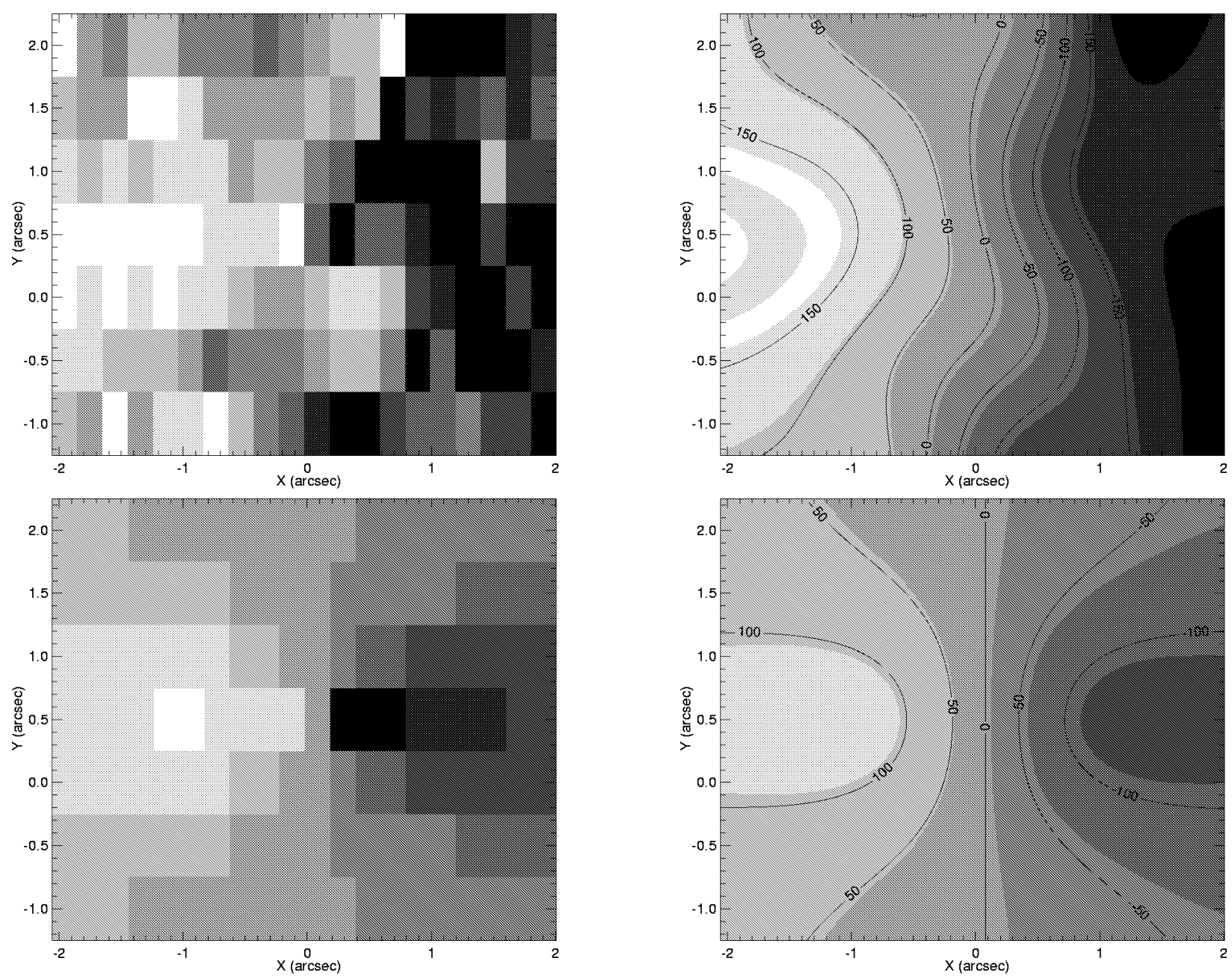

Fig. 9.- Two-dimensional velocity field of emission line gas at the off-nuclear position indicated in Figure 2. The $(x, y)$ coordinate system is centered on this position, and has the positive $x$ axis in direction the direction of $\mathrm{PA}=155$ degrees. Grey-scales run from $-225 \mathrm{~km} \mathrm{~s}^{-1}$ (black) to $+225 \mathrm{~km} \mathrm{~s}^{-1}$ (white), after subtraction of $7170 \mathrm{~km} \mathrm{~s}^{-1}$ (corresponding to the symmetry point in Figure 8a). (a; top left panel) HST/STIS measurements for individual $0.2^{\prime \prime} \times 0.5^{\prime \prime}$ "apertures". (b; bottom left panel) Model velocity field for a disk at inclination of $60^{\circ}$ rotating around a $6 \times 10^{9} \mathrm{M}_{\odot}$ black hole at $(x, y)=\left(0.1^{\prime \prime}, 0.5^{\prime \prime}\right)$. (c; top right panel) As panel (a), but smoothed with a Gaussian of dispersion 0.5". (d; top right panel) As panel (b), but smoothed with a Gaussian of dispersion $0.5^{\prime \prime}$. The model that most resembles the data has a black hole that is ten times less massive than was suggested by Bland-Hawthorn et al. (1991), and the velocity field for this model still differs considerably from the data, as discussed in the text. Combined with other evidence, this suggests that the observed velocity gradient is not due to a black hole but to the combined result of projection effects and non-equilibrium merger kinematics. 
Table 1. HST/WFPC2 images: observational setup

\begin{tabular}{|c|c|c|c|c|}
\hline (1) & $\begin{array}{c}\lambda_{0} \\
(\AA) \\
(2)\end{array}$ & $\begin{array}{c}\Delta \lambda \\
(\AA) \\
(3)\end{array}$ & $\begin{array}{c}T_{\exp } \\
\text { (sec) } \\
(4)\end{array}$ & $\begin{array}{c}N_{\exp } \\
(5)\end{array}$ \\
\hline F450W & 4410 & 925 & 2100 & 3 \\
\hline F547M & 5446 & 487 & 1200 & 3 \\
\hline F658N & 6591 & 29 & 800 & 2 \\
\hline F673N & 6732 & 47 & 2100 & 3 \\
\hline F814W & 8203 & 1758 & 1200 & 3 \\
\hline
\end{tabular}

Note. - WFPC2 images of NGC 6240 were obtained with 5 different filters. The filter name is listed in column (1). Column (2) and (3) list the central wavelength of the filter and the FWHM, as defined in Biretta et al. (2001). Column (4) lists the total exposure time per filter, which was divided over the number of exposures listed in Column (5).

Table 2. Photometric properties of nuclear components

\begin{tabular}{ccccccccc}
\hline \hline nucleus & $B$ & $V$ & $I$ & $K$ & $B-K$ & $\left\langle A_{V}\right\rangle$ & $V_{\text {corr }}$ & $\begin{array}{c}\mathrm{F}(\mathrm{H} \alpha+[\mathrm{NII}]) \\
\left(\mathrm{erg} \mathrm{cm}^{-2} \mathrm{~s}^{-1}\right)\end{array}$ \\
$(1)$ & $(2)$ & $(3)$ & $(4)$ & $(5)$ & $(6)$ & $(7)$ & $(8)$ & $(9)$ \\
\hline $\mathrm{N} 1$ & 21.00 & 19.75 & 17.73 & 14.40 & 6.60 & $2.35_{-0.33}^{+0.47}$ & 17.40 & $6.9 \times 10^{-13}$ \\
$\mathrm{~N} 2$ & 22.40 & 20.40 & 17.39 & 12.89 & 9.51 & $4.75_{-0.33}^{+0.47}$ & 15.65 & $3.7 \times 10^{-13}$ \\
$\mathrm{~N} 3$ & 21.40 & 19.58 & 17.01 & 13.22 & 8.18 & $3.65_{-0.33}^{+0.47}$ & 15.93 & $3.7 \times 10^{-13}$ \\
\hline
\end{tabular}

Note. - Column (1) is the identification of the nucleus as shown in Figure 3. Columns (2)(5) list the $B, V, I$ and $K$ magnitudes for a circular aperture with a radius of 4 pixels $\left(0.182^{\prime \prime}\right)$. Column (6) lists the implied $B-K$ color. Column (7) lists the average dust absorption $\left\langle A_{V}\right\rangle$ with an error range, determined as described in the text. Column (8) lists the estimate $V_{\text {corr }}=V-\left\langle A_{V}\right\rangle$ of the intrinsic $V$-band magnitude of each nucleus. Column (9) lists the $\mathrm{H} \alpha+[\mathrm{NII}]$ line flux of each nucleus. 